\title{
Kinetic Monte Carlo simulation of strained heteroepitaxial growth with intermixing
}

Received: 4 March 2009 / Accepted: 11 August 2009 / Published online: 24 November 2009

(C) The Author(s) 2009. This article is published with open access at Springerlink.com

\begin{abstract}
An efficient method for the simulation of strained heteroepitaxial growth with intermixing using kinetic Monte Carlo is presented. The model used is based on a solid-on-solid bond counting formulation in which elastic effects are incorporated using a ball and spring model. While idealized, this model nevertheless captures many aspects of heteroepitaxial growth, including nucleation, surface diffusion, and long-range effects due to elastic interaction. The algorithm combines a fast evaluation of the elastic displacement field with an efficient implementation of a rejection-reduced kinetic Monte Carlo based on using upper bounds for the rates. The former is achieved by using a multigrid method for global updates of the displacement field and an expanding box method for local updates. The simulations show the importance of intermixing on the growth of a strained film. Further, the method is used to simulate the growth of self-assembled stacked quantum dots.
\end{abstract}

Keywords Heteroepitaxy $\cdot$ Strained $\cdot$ Intermixing $\cdot$ Kinetic $\cdot$ Monte Carlo $\cdot$ Self-assembly

PACS 68.43.Jk $\cdot 68.65 . \mathrm{Hb} \cdot 68.55 .-\mathrm{a} \cdot 81.16 \mathrm{Dn} \cdot 81.15 . \mathrm{Aa}$

\section{Introduction}

Heteroepitaxy is the process of slow deposition of a film of one or more crystalline materials on a crystalline substrate of a different material. The classical examples of film/substrate combinations include, for example $\mathrm{Ge} / \mathrm{Si}$, InAs/GaAs, and InP/GaAs. The natural lattice spacing between the film and the substrate differ by a few percent resulting in elastic stress. Consequently, as the film grows the elastic energy builds up. The formation of 3D islands reduces the elastic energy by relieving stress at the cost of increased surface energy. In many cases these islands are on the order of tens of nanometers and are referred to as quantum dots. These quantum dot materials are of importance in construction of some optoelectronic devices.

The theory of island formation is well understood in the context of Asaro-Tiller-Grinfeld instability $[1,6]$. This explains the instability of a single component stressed film to perturbations of the flat surface. The flat surface is unstable under sufficiently long wavelength perturbations. These perturbations grow through mass

Communicated by R. Caflisch.

A. Baskaran $(\varangle) \cdot$ P. Smereka

Department of Mathematics, University of Michigan, Ann Arbor, MI, USA

E-mail: baskaran@umich.edu

P. Smereka

E-mail: psmereka@umich.edu

J. Devita

Department of Mathematics, University of California, Los Angeles, CA, USA

E-mail: jason@math.ucla.edu 
transport on the surface, along the free energy gradients, leading to formation of surface ripples that later grow into large islands with stress concentration in the valleys. The theory, however, fails to explain an experimental observation, namely in many cases the film first grows in a layer-by-layer fashion until it reaches a certain critical thickness. Then islands form on top of this layer (known as the wetting layer). This mode of growth is known as the Stranski-Krastonov (SK) growth mode $[13,18]$.

It was demonstrated experimentally by Cullis et al. [25,5], that the intermixing between the film and substrate is of importance. The presence of compositional non-uniformity and the dilution of the film by substrate material can have a stabilizing effect. Moreover, the presence of vertical and lateral segregation in the case of alloy films [24], can further change the nature of the instability. Subsequently, Tu and Tersoff [22], using a continuum model developed by Spencer etal. [21], argued that Stranski-Krastonov growth is a kinetic effect and the wetting layer is not stable. A crucial aspect of their proposal is the intermixing that occurs between the film and the substrate.

Another important example in which intermixing occurs on very large length scales is the self assembly of stacked quantum dots. Here, the dominant mechanism of intermixing results from the deposition process in which several layers of one material deposited which are followed by several layers of another material and so on. Since the materials are not lattice matched elastic strain develops and it is observed that the film forms quantum dots. The quantum dots in different layers align themselves to self assemble into stacked quantum dots. Intermixing of the film material and the capping layer needs to be addressed in this situation. Some related study, both experimental and computational, can be found in References [23,10,9,11,27,14].

It is clear that the simulation of strained heteroepitaxial growth with intermixing has important applications. One reasonably popular approach is based on numerical solution of continuum equations. Indeed, recent study in this direction [21-23,26,27] has been able to capture many aspects of the film growth. Another approach is based on kinetic Monte Carlo (KMC). Since, KMC is based on an atomistic scale it is typically slower than a continuum formulation. On the other hand, KMC not only captures all the physical effects modeled by continuum approach but can also naturally include discrete and stochastic effects such as nucleation, surface roughness, and intermixing.

The purpose of this article is to present a KMC model of strained heteroepitaxial growth with intermixing and an algorithm for its efficient simulation. We extended the model proposed by Orr et al. [12] and Lam etal. [7] to include intermixing. This model is a solid-on-solid bond counting scheme [19] in which elastic interactions are included using a ball and spring model. The efficient simulation of this model represents the major contribution of this article.

One computational bottle neck for the simulation of strained film growth is the repeated calculation of elastic field. In this article, we address this by the inclusion of intermixing into the multigrid-Fourier method developed by Russo and Smereka $[15,16]$. In addition, we present a cleaner way to incorporate the substrate into the multigrid method and simplify the coarse-graining and prolongation operators. As it turns out, for the KMC model used here, the change in total elastic energy, when a surface atom is removed, is needed. It was established by Schulze and Smereka [17] that despite the long-range nature of elastic interactions one can accurately compute this energy change by local calculations. In this study, we have verified that this approach also works in case of intermixing. In addition, we have presented numerical evidence that the asymptotic expressions presented in Ref. [17] remain valid in the case of intermixing.

Another computational bottleneck is the computation of the rates. In order to implement rejection-free KMC one must know the hopping rates for all the atoms. In order to accomplish this, one would have to compute the change in elastic energy that occurs when each and every atom is removed. This would be prohibitively expensive. Fortunately, it was argued in Ref. [17] that the current elastic energy density can be used to provide fairly sharp upper bounds on the change in elastic energy. These can be used to provide a rejection-reduced KMC algorithm. Here, we demonstrate that this approach works well for the intermixing case. In our simulations the rejection rate is approximately between .5\% and 5\%.

In the following sections, we explain the KMC model and present our algorithm to calculate the elastic displacement field and elastic energy in the case of a multicomponent film. Some results are presented that illustrate the effectiveness of the algorithm; namely, the effects of intermixing on the morphology of a growing strained film and a simulation illustrating the self-assembly of stacked quantum dots. To the best of our knowledge these results are the first kinetic Monte Carlo simulations of strained heteroepitaxial growth with intermixing. 


\section{Model description}

Our model is based on the solid-on-solid model presented by Orr et al. [12] and Lam et al. [7]. We consider a semi-infinite substrate which is initially composed of a single component. For ease of exposition, we shall refer to the substrate material as Silicon. The deposited material will be composed of a prescribed mixture of Silicon and Germanium. The model and algorithm will be detailed for a two component film but it is not limited to this. In this article, we restrict ourselves to $1+1$ dimensions. The atoms in the crystal occupy sites on a simple cubic crystal, within the solid-on-solid framework (no overhanging atoms). Therefore, the height of the surface is a function of the horizontal coordinate denoted by $\ell$. Each atom in the lattice is bonded with its nearest (four possible) and next to nearest neighbors (four possible) by bonds of strength $\gamma$.

The elastic interactions are modeled by means of a ball and spring system. Each atom on the lattice is connected to its nearest and next to nearest neighbors by Hookean springs. The lateral and vertical springs have a spring constant of $k_{\mathrm{L}}$ and the diagonal springs $k_{\mathrm{D}}$. We choose $k_{\mathrm{D}}=k_{\mathrm{L}} / 2$ corresponding to the isotropic case [7]. The natural bond lengths (lattice spacing) are denoted by $a_{\mathrm{ss}}, a_{\mathrm{sg}}$ and $a_{\mathrm{gg}}$, for $\mathrm{Si}-\mathrm{Si}, \mathrm{Si}-\mathrm{Ge}$ and $\mathrm{Ge}-\mathrm{Si}$ bonds, respectively. If these quantities are equal there are no forces due to lattice mismatch and hence no elastic energy in the crystal. In general, since $a_{\mathrm{ss}} \neq a_{\mathrm{sg}} \neq a_{\mathrm{gg}}$ forces do arise. This is addressed in detail in the next section.

The crystal evolves by rearranging itself through motion of surface atoms. By virtue of the solid-on-solid assumption each surface atom is uniquely specified by $\ell$ (the horizontal component) and this is what we mean when we specify the $\ell$ th surface atom. The hopping rate of the $\ell$ th surface atom is modeled by

$$
R_{\ell}=R_{0} \exp \left[\frac{\Delta E+E_{0}}{k_{\mathrm{B}} T}\right]
$$

where $\Delta E$ is the change in the total energy when removing the $\ell$ th surface atom, $R_{0}$ is the attempt frequency, $k_{\mathrm{B}}$ is the Boltzmann constant and $T$ is the absolute temperature. We write the total energy as

$$
E=E_{\text {chem }}+W
$$

where $E_{\text {chem }}$ is the total chemical energy and $W$ is the total elastic energy. The chemical energy can be thought of as the contribution to the total energy from local interactions whereas $W$ is the contribution by the long-range interactions. Since, we are assuming that the bond energy is the same for nearest and next to nearest neighbors, it follows that

$$
\Delta E_{\text {chem }}=-\gamma N
$$

where $N$ is the total number of nearest and next to nearest neighbors. Therefore, the change in total energy is given by

$$
\Delta E=-\gamma N+\Delta W
$$

The calculation of $\Delta W$ is described in the next two sections. The parameters $E_{0}$ and $R_{0}$ will be chosen to match the adatom diffusion rate to experimental values. When an atom hops it moves to another surface site whose horizontal coordinate changes by \pm 1 , with equal probability. It should be noted that since only surface atoms can move in this model intermixing can only arise due to surface roughness combined with adatom diffusion.

As a simplification, we shall ignore the elastic contribution to the energy barrier for adatoms; therefore, the rates, we shall use are

$$
R_{\ell}= \begin{cases}R_{0} \exp \left(\frac{-3 \gamma+E_{0}}{k_{\mathrm{B}} T}\right) & \text { if } N \leq 3 \\ R_{0} \exp \left(\frac{-N \gamma+\Delta W+E_{0}}{k_{\mathrm{B}} T}\right) & \text { if } N>3\end{cases}
$$

The number 3 corresponds to the number of bonds of an adatom on a flat terrace. The adatoms with less than 3 bonds are assumed to hop at the same rate as other adatoms. The change in elastic energy when a surface atom is removed is usually a small fraction of the change in chemical energy. Nevertheless, these small changes become important when their variation is due the morphology of the surface. We have found, however, that 
the change in elastic energy when removing an adatom is insensitive to the surface morphology. In other words, the adatom hops merely serve as a mechanism for surface mass transport. Based on this, we do not consider the contribution of $\Delta W$ in the hopping rate of adatoms. Numerical studies confirm that there is no significant change in the results due to this treatment for the adatoms, however, it reduces the computational time significantly. This was also reported by [7].

Finally, we deposit atoms at a rate of

$$
R_{\mathrm{dep}}=F M
$$

where $F$ is the deposition rate in monolayers per unit time and $M$ is the total number of sites in the horizontal direction.

The model by Lam et al. [7] allowed the possibility of large hops with $R_{0}$ adjusted to correct for the large hop size. This greatly improved the computational speed but at the same time not changing the results too much. However, in the case of intermixing we observed that the inclusion of this feature significantly retarded intermixing. Hence, large hop strategy was not adopted in this study.

\section{The displacement field}

The calculation of the hopping rate of an atom involves the computation of elastic energy of the crystal in different configurations. The elastic energy is merely the sum total of the energy stored in each spring when the crystal is in mechanical equilibrium. The energy in a harmonic spring is proportional to the square of the change in length of the spring (relative to natural length). This is computed in terms of the displacements of the atoms from a reference configuration. The computational challenge is in quickly computing the equilibrium displacements of the atoms.

\subsection{Forces in the reference configuration}

The first step toward the calculation of the elastic energy is the choice of a reference configuration. The displacements of atoms are calculated with respect to this reference configuration. We choose this to be a cubic lattice, whose lattice spacing equals that of pure substrate material. This ensures that the substrate is devoid of any forces in the reference state. Thus, two atoms in the reference configuration are a distance $a_{\mathrm{ss}}$ apart. The natural lattice spacing, however, depends on the nature of the two atoms. The natural lattice spacing is $a=a_{\mathrm{ss}}, a_{\mathrm{gs}}$, and $a_{\mathrm{gg}}$, when the two atoms are $\mathrm{Si}-\mathrm{Si}, \mathrm{Ge}-\mathrm{Si}$, and $\mathrm{Ge}-\mathrm{Ge}$ respectively. It is useful to introduce the misfit parameter, $\epsilon$,

$$
\epsilon=\frac{\left(a-a_{\mathrm{ss}}\right)}{a_{\mathrm{ss}}}
$$

This typically varies from $\epsilon=-0.06$ to +0.06 . In the two component system $\mathrm{Ge} / \mathrm{Si}$, we have two interactions to be considered $\mathrm{Ge}-\mathrm{Si}$ and $\mathrm{Ge}-\mathrm{Ge}$ and we introduce the following quantities

$$
\epsilon_{\mathrm{sg}}=\frac{\left(a_{\mathrm{sg}}-a_{\mathrm{ss}}\right)}{a_{\mathrm{ss}}} \text { and } \epsilon_{\mathrm{gg}}=\frac{\left(a_{\mathrm{gg}}-a_{\mathrm{ss}}\right)}{a_{\mathrm{ss}}} .
$$

In order to compute the forces, that arise from the misfit, on the atoms in the reference configuration let us focus on two nearest neighbor atoms. The force exerted by the spring on the atoms can be calculated to be $k_{\mathrm{L}}\left(a-a_{\mathrm{ss}}\right)$ in magnitude where $k_{\mathrm{L}}$ is the spring constant for nearest neighbor atoms. In the case of next to nearest neighbor interaction, the force is calculated to be $k_{\mathrm{D}}\left(a-a_{\mathrm{ss}}\right)$ in magnitude, to first order in $\epsilon$. The spring constant for next to nearest neighbors is $k_{\mathrm{D}}$. Before the general formula for the forces in the reference configuration are given, we first present a simple example.

An Example. In order to make things clear, we present a sample calculation of forces in the configuration shown in Fig. 1. The forces are given by

$$
\mathbf{F}^{-1,1}=\left(\begin{array}{l}
0 \\
0
\end{array}\right), \quad \mathbf{F}^{0,1}=\left(\begin{array}{l}
0 \\
0
\end{array}\right), \quad \text { and } \quad \mathbf{F}^{1,1}=\left(\begin{array}{c}
-F_{N N}^{\mathrm{gg}} \\
-F_{N N}^{\mathrm{gg}}
\end{array}\right) .
$$




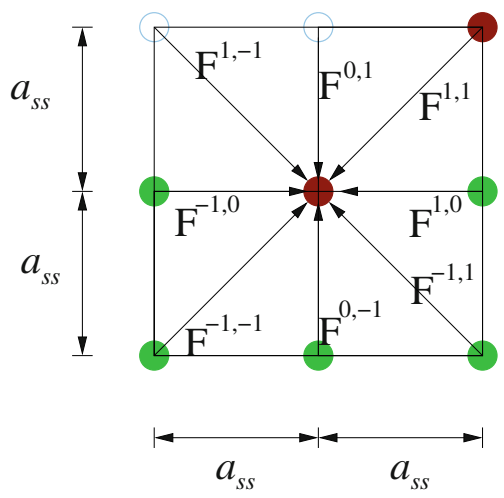

Fig. 1 Configuration shows the atom of interest in the center surrounded by eight neighboring sites. The Green circles represent $\mathrm{Si}$ atoms, the red represent $\mathrm{Ge}$ and blue the empty sites.

The first two correspond to interactions with empty sites and the last one arises from a Ge-Ge interaction. The forces from $\mathrm{Si}-\mathrm{Ge}$ interactions are given by

$$
\begin{aligned}
& \mathbf{F}^{-1,0}=\left(\begin{array}{c}
F_{N}^{\mathrm{sg}} \\
0
\end{array}\right), \quad \mathbf{F}^{1,0}=\left(\begin{array}{c}
-F_{N}^{\mathrm{sg}} \\
0
\end{array}\right), \\
& \mathbf{F}^{0,-1}=\left(\begin{array}{c}
0 \\
F_{N}^{\mathrm{sg}}
\end{array}\right), \quad \mathbf{F}^{-1,-1}=\left(\begin{array}{c}
F_{N N}^{\mathrm{sg}} \\
F_{N N}^{\mathrm{sg}}
\end{array}\right), \quad \mathbf{F}^{1,-1}=\left(\begin{array}{c}
F_{N N}^{\mathrm{sg}} \\
-F_{N N}^{\mathrm{sg}}
\end{array}\right) .
\end{aligned}
$$

where

$$
F_{N}^{\mathrm{sg}}=k_{\mathrm{L}} \epsilon_{\mathrm{sg}} a_{\mathrm{ss}}, \quad F_{N N}^{\mathrm{sg}}=k_{\mathrm{D}} \epsilon_{\mathrm{sg}} a_{\mathrm{ss}}, \quad \text { and } \quad F_{N N}^{\mathrm{gg}}=k_{\mathrm{D}} \epsilon_{\mathrm{gg}} a_{\mathrm{ss}}
$$

The General Case. The crystal is a lattice indexed by $(\ell, j)$. The first index represents the horizontal coordinate. The crystal is periodic in this index. The second is the vertical coordinate. The crystal is semi-infinite in this index, i.e., $j<h_{\ell}$ where $h_{\ell}$ is the location of the surface. All sites $j>h_{\ell}$ are unoccupied.

We now write down the misfit forces for the general case. The net force on an atom at site $(\ell, j)$ in the reference state, due to its nearest and next to nearest neighbors $(j+m, \ell+n)$ where $(m, n) \in$ $(\{-1,0,1\},\{-1,0,1\})$, is given by

$$
\left(\begin{array}{c}
F_{\ell j} \\
G_{\ell j}
\end{array}\right)=\sum_{m, n=-1,0,1} \mathbf{F}_{\ell j}^{m n}
$$

where $\mathbf{F}_{\ell j}^{m n}$ are given by

$$
\begin{aligned}
\mathbf{F}_{\ell j}^{-1,1}=\left(\begin{array}{c}
f_{\ell, j}^{-1,1} \\
f_{\ell, j}^{-1,1}
\end{array}\right) \quad \mathbf{F}_{\ell j}^{0,1}=\left(\begin{array}{c}
0 \\
f_{\ell, j}^{0,1}
\end{array}\right) \quad \mathbf{F}_{\ell j}^{1,1}=\left(\begin{array}{c}
f_{\ell, j}^{1,1} \\
-f_{\ell, j}^{1,1}
\end{array}\right) \\
\mathbf{F}_{\ell j}^{-1,0}=\left(\begin{array}{c}
f_{\ell, j}^{-1,0} \\
0
\end{array}\right) \\
\mathbf{F}_{\ell j}^{1,0}=\left(\begin{array}{c}
-f_{\ell, j}^{1,0} \\
0
\end{array}\right) \\
\mathbf{F}_{\ell j}^{-1,-1}=\left(\begin{array}{c}
f_{\ell, j}^{-1,-1} \\
f_{\ell, j}^{-1,-1}
\end{array}\right) \mathbf{F}_{\ell j}^{0,-1}=\left(\begin{array}{c}
0 \\
f_{\ell, j}^{0,-1}
\end{array}\right) \mathbf{F}_{\ell j}^{1,-1}=\left(\begin{array}{c}
f_{\ell, j}^{1,-1} \\
-f_{\ell, j}^{1,-1}
\end{array}\right),
\end{aligned}
$$

with $f_{\ell j}^{m n}$ defined as

$$
f_{\ell j}^{m n}= \begin{cases}\sigma_{i, j ; n, m} \epsilon_{i, j ; m, n} k_{\mathrm{D}} a_{\mathrm{SS}} & \text { if }(m, n) \in( \pm 1, \pm 1) \\ \sigma_{i, j ; n, m} \epsilon_{i, j ; m, n} k_{\mathrm{L}} a_{\mathrm{ss}} & \text { otherwise. }\end{cases}
$$


The connectivity matrix, $\sigma_{i, j ; m, n}$, is defined as

$$
\sigma_{\ell, j ; n, m}= \begin{cases}1 & \text { if sites }(\ell, j) \text { and }(\ell+m, j+n) \text { both contain atoms } \\ 0 & \text { otherwise }\end{cases}
$$

and the misfit matrix, $\epsilon_{\ell, j ; n, m}$, is defined as

$$
\epsilon_{\ell, j ; n, m}= \begin{cases}\epsilon_{\mathrm{gg}} & \text { if sites }(\ell, j) \text { and }(\ell+m, j+n) \text { both contain Ge atoms } \\ \epsilon_{\mathrm{gg}} & \text { if sites }(\ell, j) \text { and }(\ell+m, j+n) \text { contain a Ge and a Si atom } \\ 0 & \text { otherwise }\end{cases}
$$

Note: The misfit for a Ge/Si system is $\epsilon_{\mathrm{gg}}=0.04$ is known. However, our model demands a parameter $\epsilon_{\mathrm{sg}}$ which is not clear. We have conducted simulations with both $\epsilon_{\mathrm{sg}}=0.04$ and $\epsilon_{\mathrm{sg}}=.02$. The first choice considers $a_{\mathrm{sg}}=a_{\mathrm{gg}}$ and the second considers $a_{\mathrm{sg}}=0.5 a_{\mathrm{gg}}$. The second choice seems appropriate if one considers the two species as hard spheres of different sizes. Preliminary simulations with the first choice revealed quantitative differences in the island sizes but no apparent qualitative differences in the nature of the instability. With the true nature of the interactions being unknown, we present only results corresponding to $\epsilon_{\mathrm{sg}}=0.02$.

\subsection{Interactions}

The atoms in the reference state experience forces from their neighbors as given by Eq. 10. The atoms become displaced from the reference position to achieve mechanical equilibrium (force balance) and we denote the displacement of the atom at site $(\ell, j)$ as $\left(u_{\ell j}, v_{\ell j}\right)^{T}$.

Our aim is to calculate $\left(u_{\ell j}, v_{\ell j}\right)^{T}$. We will assume that there is a $J$ such that the crystal is made of pure $\mathrm{Si}$ for $j \leq J$ and that all sites in this region are occupied. This means that in the region $j \leq J$ the misfit forces given by Eq. 10 will be zero. Whereas these forces will be nonzero for $j \geq J$; this includes the deposited atoms and the regions where the deposited atoms have intermixed with the substrate atoms. We treat these two regions separately. In order to set a convenient language for the rest of this article, we will refer to these regions as the film $(j \geq J)$ and substrate $(j<J)$, not to be confused with the original substrate and the deposited material. Further, we choose our indices such that $J=0$ for convenience.

\subsubsection{Interactions in the film region}

For the film region corresponding to the indices $j \geq 0$, the force balance gives us

$$
\begin{aligned}
0= & F_{\ell j}+k_{\mathrm{L}}\left(\sigma_{\ell, j ; 1,0}\left(u_{\ell+1, j}-u_{\ell j}\right)+\sigma_{\ell, j ;-1,0}\left(u_{\ell-1, j}-u_{\ell j}\right)\right) \\
& +\frac{k_{\mathrm{D}}}{2}\left(\sigma_{\ell, j ; 1,1}\left(u_{\ell+1, j+1}-u_{\ell j}\right)+\sigma_{\ell, j ;-1,1}\left(u_{\ell-1, j+1}-u_{\ell j}\right)\right) \\
& +\frac{k_{\mathrm{D}}}{2}\left(\sigma_{\ell, j ; 1,-1}\left(u_{\ell+1, j-1}-u_{\ell j}\right)+\sigma_{\ell, j ;-1,-1}\left(u_{\ell-1, j-1}-u_{\ell j}\right)\right) \\
& +\frac{k_{\mathrm{D}}}{2}\left(\sigma_{\ell, j ; 1,1}\left(v_{\ell+1, j+1}-v_{\ell j}\right)-\sigma_{\ell, j ;-1,1}\left(v_{\ell-1, j+1}-v_{\ell j}\right)\right) \\
& +\frac{k_{\mathrm{D}}}{2}\left(-\sigma_{\ell, j ; 1,-1}\left(v_{\ell+1, j-1}-v_{\ell j}\right)+\sigma_{\ell, j ;-1,-1}\left(v_{\ell-1, j-1}-v_{\ell j}\right)\right)
\end{aligned}
$$

and

$$
\begin{aligned}
0= & G_{\ell j}+k_{\mathrm{L}}\left(\sigma_{\ell, j ; 0,1}\left(v_{\ell j+1}-v_{\ell j}\right)+\sigma_{\ell, j ; 0,-1}\left(v_{\ell, j-1}-v_{\ell j}\right)\right) \\
& +\frac{k_{\mathrm{D}}}{2}\left(\sigma_{\ell, j ; 1,1}\left(v_{\ell+1, j+1}-v_{\ell j}\right)+\sigma_{\ell, j ;-1,1}\left(v_{\ell-1, j+1}-v_{\ell j}\right)\right) \\
& +\frac{k_{\mathrm{D}}}{2}\left(\sigma_{\ell, j ; 1,-1}\left(v_{\ell+1, j-1}-v_{\ell j}\right)+\sigma_{\ell, j ;-1,-1}\left(v_{\ell-1, j-1}-v_{\ell j}\right)\right) \\
& +\frac{k_{\mathrm{D}}}{2}\left(\sigma_{\ell, j ; 1,1}\left(u_{\ell+1, j+1}-u_{\ell j}\right)-\sigma_{\ell, j ;-1,1}\left(u_{\ell-1, j+1}-u_{\ell j}\right)\right) \\
& +\frac{k_{\mathrm{D}}}{2}\left(-\sigma_{\ell, j ; 1,-1}\left(u_{\ell+1, j-1}-u_{\ell j}\right)+\sigma_{\ell, j ;-1,-1}\left(u_{\ell-1, j-1}-u_{\ell j}\right)\right)
\end{aligned}
$$


where $\sigma_{\ell, j ; n, m}$ is the connectivity matrix defined in Eq. 13. This gives a linear system of equations that can be solved for $\left(u_{\ell j}, v_{\ell j}\right)$. This system interacts with the substrate region. Note that the above relations at $j=0$ use $u_{\ell,-1}, v_{\ell,-1}$ which are displacements in the substrate.

\subsubsection{Interactions in the substrate region}

Now for the region $j<0$, we first note that, this is a semi-infinite substrate. There are no forces arising from misfit in the substrate and requiring mechanical equilibrium gives us

$$
\begin{aligned}
0= & k_{\mathrm{L}}\left(u_{\ell+1, j}-2 u_{\ell, j}+u_{\ell-1, j}\right) \\
& +\frac{k_{\mathrm{D}}}{2}\left(u_{\ell+1, j+1}+u_{\ell-1, j+1}+u_{\ell+1, j-1}+u_{\ell-1, j-1}-4 u_{\ell j}\right) \\
& +\frac{k_{\mathrm{D}}}{2}\left(v_{\ell+1, j+1}+v_{\ell-1, j-1}-v_{\ell+1, j-1}-v_{\ell-1, j+1}\right) \\
0= & k_{\mathrm{L}}\left(v_{\ell j+1}-2 v_{\ell j}+v_{\ell j-1}\right) \\
& +\frac{k_{\mathrm{D}}}{2}\left(v_{\ell+1, j+1}+v_{\ell-1, j+1}+v_{\ell+1, j-1}+v_{\ell-1, j-1}-4 v_{\ell, j}\right) \\
& +\frac{k_{\mathrm{D}}}{2}\left(u_{\ell+1, j+1}+u_{\ell-1, j-1}-u_{\ell+1, j-1}-u_{\ell-1, j+1}\right) .
\end{aligned}
$$

This is a homogeneous system of equations that takes a non-trivial solution due to the displacement field of the film (at $j=0$ ). This gives us the displacement field for $j<0$ of a relaxed substrate with given displacements at $j=0$. The system in Eqs. 17 and 18 needs to be solved for $j<0$ given $\left(u_{\ell, 0}, v_{\ell, 0}\right)$. We do this by using a Fourier series in the $x$-direction:

$$
\left(\begin{array}{c}
u_{\ell j} \\
v_{\ell j}
\end{array}\right)=\sum_{\xi=1}^{M}\left(\begin{array}{c}
\widehat{u}_{\xi j} \\
\widehat{v}_{\xi j}
\end{array}\right) e^{i \ell \xi} .
$$

Now, inserting this into Eqs. 17 and 18 we get

$$
\begin{aligned}
0= & 2 k_{\mathrm{L}} \widehat{u}_{\xi, j}(\cos \xi-1)+k_{\mathrm{D}}\left[\left(\widehat{u}_{\xi, j-1}+\widehat{u}_{\xi, j+1}\right) \cos \xi-2 \widehat{u}_{\xi j}\right. \\
& \left.+i\left(\widehat{v}_{\xi, j+1}-\widehat{v}_{\xi, j-1}\right) \sin \xi\right] \\
0= & k_{\mathrm{L}}\left(\widehat{v}_{\xi, j+1}-2 \widehat{v}_{\xi j}+\widehat{v}_{\xi, j-1}\right)+k_{\mathrm{D}}\left[\left(\widehat{v}_{\xi, j-1}+\widehat{v}_{\xi, j-1}\right) \cos \xi-2 \widehat{v}_{\xi, j}\right. \\
& \left.+i\left(\widehat{u}_{\xi, j+1}-\widehat{u}_{\xi, j-1}\right) \sin \xi\right] .
\end{aligned}
$$

We look for a solution for the above system in the form

$$
\hat{u}_{\xi j}=\alpha^{j} \hat{u}_{\xi, 0}, \quad \hat{v}_{\xi, j}=\alpha^{j} \hat{v}_{\xi, 0}
$$

and obtain a linear homogeneous system of the form

$$
\boldsymbol{\Omega}(\alpha)\left(\begin{array}{c}
\hat{u}_{\xi, 0} \\
\hat{v}_{\xi, 0}
\end{array}\right)=0 .
$$

The above system admits non-trivial solutions if

$$
P(\alpha):=\operatorname{det}[\Omega(\alpha)]=0
$$

giving raise to a polynomial equation in $\alpha$. The polynomial $P(\alpha)$ is of degree 4 and has roots of the form $\left(\alpha_{1}, \alpha_{2}, 1 / \alpha_{1}, 1 / \alpha_{2}\right)$ where $\left|\alpha_{1}\right|,\left|\alpha_{2}\right|>1$ [15]. Since, we are interested in solutions $\hat{u}_{j}=\alpha^{j} \hat{u}_{0}, \quad \hat{v}_{j}=\alpha^{j} \hat{v}_{0}$ that decay as $j \rightarrow-\infty$ we pick the two roots $\alpha_{1}, \alpha_{2}$.

$$
\left(\begin{array}{c}
\widehat{u}_{\xi, j} \\
\widehat{v}_{\xi, j}
\end{array}\right)=Q(j) Q^{-1}(0)\left(\begin{array}{c}
\widehat{u}_{\xi, 0} \\
\widehat{v}_{\xi, 0}
\end{array}\right)
$$


where $Q(j)$ is the invertible $2 \times 2$ matrix given by

$$
\left(\begin{array}{ll}
\mathbf{r}_{1} \alpha_{1}^{j} & \mathbf{r}_{2} \alpha_{2}^{j}
\end{array}\right)
$$

Finally, $\mathbf{r}_{\mathbf{p}}$ and $\alpha_{p}$ are the eigenvectors and eigenvalues that arise when solving the discrete equation. The solution of Eqs. (17) and (18) is then given by

$$
\left(\begin{array}{c}
u_{\ell, j} \\
v_{\ell, j}
\end{array}\right)=\sum_{\xi=1}^{M} Q(j) Q^{-1}(0)\left(\begin{array}{c}
\widehat{u}_{\xi 0} \\
\widehat{v}_{\xi 0}
\end{array}\right) e^{i \ell \xi} \text { where }\left(\begin{array}{c}
\widehat{u}_{\xi 0} \\
\widehat{v}_{\xi 0}
\end{array}\right)=\frac{1}{M} \sum_{\xi=1}^{M}\left(\begin{array}{c}
u_{\ell, 0} \\
v_{\ell, 0}
\end{array}\right) e^{-i \xi \ell}
$$

A three dimensional version of this was presented in [15] and similar approach was presented in [8].

\section{Elastic energy of the crystal}

The elastic energy can be computed using the displacement field calculated by the algorithm in the previous section; it is

$$
W=\sum_{\text {all springs }} \frac{1}{2} k_{\text {spring }} \delta^{2}
$$

where $k_{\text {spring }}=k_{\mathrm{L}}, k_{\mathrm{D}}$ depending on whether the spring is a diagonal or lateral spring, and $\delta$ is the change in spring length with respect to natural spring length. We rewrite $W$ as

$$
W=W_{\mathrm{f}}+W_{\mathrm{s}}
$$

where

$$
W_{\mathrm{f}}=\sum_{j>0} \frac{1}{2} k_{\mathrm{spring}} \delta^{2} \quad \text { and } \quad W_{\mathrm{s}}=\sum_{j \leq 0} \frac{1}{2} k_{\mathrm{spring}} \delta^{2}
$$

where $\sum_{j>0}$ indicates summing all springs connected to atoms located at sites with $j>0, \sum_{j \leq 0}$ is defined in a similar way. We can write the expression of $W_{\mathrm{f}}$ as a sum over atoms:

$$
W_{\mathrm{f}}=\frac{1}{2} \sum_{j \geq 0} w_{i, j}
$$

where $w_{i, j}$ is the energy of all the springs connected to the atom at site $(i, j)$. The expression for $w_{i, j}$ is given at the end of this section. Since, there are no forces arising from misfit in the substrate we can write the expression for the elastic energy of the substrate

$$
W_{\mathrm{s}}=-\frac{1}{2} \mathbf{u}_{\mathrm{s}}^{T} \mathbf{A}_{\mathrm{s}} \mathbf{u}_{\mathrm{s}}
$$

where $\mathbf{A}_{\mathrm{s}}$ is the non-positive infinite dimensional matrix representing the interaction of the atoms in the substrate and $\mathbf{u}_{\mathrm{s}}$ is the vector representing their displacements. It is convenient to decompose $\mathbf{u}_{\mathrm{s}}$ into the displacements of the atoms at $j=0$ and those for $j<0$ :

$$
\mathbf{u}_{s}=\left(\begin{array}{l}
\mathbf{u}_{0} \\
\mathbf{u}_{j<0}
\end{array}\right)
$$

Since atoms in the substrate below the first layer produce no net force on each other it follows

$$
\mathbf{A}_{\mathrm{s}}\left(\begin{array}{l}
\mathbf{u}_{0} \\
\mathbf{u}_{j<0}
\end{array}\right)=\left(\begin{array}{l}
\mathbf{f}_{0} \\
0
\end{array}\right)
$$


where $\mathbf{f}_{0}$ is the force on the top layer of the substrate atoms due to the substrate atoms. If we use the above expression in the equation for $W_{s}$ we find

$$
W_{\mathrm{s}}=-\frac{1}{2} \mathbf{f}_{0} \cdot \mathbf{u}_{0}
$$

which can be written as

$$
W_{\mathrm{s}}=-\frac{1}{2} \sum_{\ell=1}^{M}\left(u_{\ell, 0} f_{\ell}+v_{\ell, 0} g_{\ell}\right)
$$

where

$$
f_{\ell}=k_{\mathrm{L}}\left(u_{\ell-1,0}-2 u_{\ell, 0}+u_{\ell+1}\right)+\frac{k_{\mathrm{D}}}{2}\left(u_{\ell-1,-1}-2 u_{\ell, 0}+u_{\ell+1,-1}\right)+\frac{k_{\mathrm{D}}}{2}\left(v_{\ell-1,-1}-v_{\ell+1,-1}\right)
$$

and

$$
g_{\ell}=k_{\mathrm{L}}\left(-v_{\ell, 0}+v_{\ell,-1}\right)+\frac{k_{\mathrm{D}}}{2}\left(v_{\ell-1,-1}-2 v_{\ell, 0}+v_{\ell+1,-1}\right)+\frac{k_{\mathrm{D}}}{2}\left(u_{\ell-1,-1}-u_{\ell+1,-1}\right) .
$$

Therefore, the total energy of the crystal is given by Eq. 25 along with Eqs. 26 and 27). It should be pointed out that this expression could have also been obtained using summation by parts. Finally, as promised we present the expression for $w_{i, j}$

$$
w_{i, j}=w_{i, j}^{x x}+w_{i, j}^{y y}+2 w_{i, j}^{x y}
$$

with

$$
\begin{aligned}
w_{i j}^{x x}= & \frac{k_{\mathrm{L}}}{2}\left(\sigma_{i, j ; 1,0}\left(u_{i+1, j}-u_{i, j}-d\right)^{2}+\sigma_{i, j ;-1,0}\left(u_{i-1, j}-u_{i, j}+d\right)^{2}\right) \\
& +\frac{k_{\mathrm{D}}}{4}\left(\sigma_{i, j ; 1,1}\left(u_{i+1, j+1}-u_{i, j}-d\right)^{2}+\sigma_{i, j ;-1,-1}\left(u_{i-1, j-1}-u_{i, j}+d\right)^{2}\right. \\
& \left.+\sigma_{i, j ; 1,-1}\left(u_{i+1, j-1}-u_{i, j}-d\right)^{2}+\sigma_{i, j ;-1,1}\left(u_{i-1, j+1}-u_{i, j}+d\right)^{2}\right), \\
w_{i j}^{y y}= & \frac{k_{\mathrm{L}}}{2}\left(\sigma_{i, j ; 0,1}\left(v_{i, j+1}-v_{i, j}-d\right)^{2}+\sigma_{i, j ; 0,-1}\left(v_{i, j-1}-v_{i, j j}+d\right)^{2}\right) \\
+ & \frac{k_{\mathrm{D}}}{4}\left(\sigma_{i, j ; 1,1}\left(v_{i+1, j+1}-v_{i, j}-d\right)^{2}+\sigma_{i, j ;-1,-1}\left(v_{i-1, j-1}-v_{i, j}+d\right)^{2}\right. \\
+ & \left.\sigma_{i, j ; 1,-1}\left(v_{i+1, j-1}-v_{i, j}+d\right)^{2}+\sigma_{i, j ;-1,1}\left(v_{i-1, j+1}-v_{i, j}-d\right)^{2}\right), \\
w_{i j}^{x y}= & \frac{k_{\mathrm{D}}}{4}\left(\sigma_{i, j ;-1,-1}\left(u_{i-1, j-1}-u_{i, j}+d\right)\left(v_{i-1, j-1}-v_{i, j}+d\right)\right. \\
& +\sigma_{i, j ; 1,1}\left(u_{i+1, j+1}-u_{i, j}-d\right)\left(v_{i+1, j+1}-u_{i, j}-d\right) \\
& -\sigma_{i, j ; 1,-1}\left(u_{i+1, j-1}-u_{i, j}-d\right)\left(v_{i+1, j-1}-v_{i, j}+d\right) \\
& \left.-\sigma_{i, j ;-1,1}\left(u_{i-1, j+1}-u_{i, j}+d\right)\left(v_{i-1, j+1}-v_{i, j}-d\right)\right),
\end{aligned}
$$

where

$$
d=\left\{\begin{array}{cl}
a_{\mathrm{gs}}-a_{\mathrm{ss}} & \mathrm{Ge}-\mathrm{Si} \text { bonds } \\
a_{\mathrm{gg}}-a_{\mathrm{ss}} & \mathrm{Ge}-\mathrm{Ge} \text { bonds } \\
0 & \text { for Si-Si bonds }
\end{array}\right.
$$




\section{Multigrid Fourier algorithm}

In this section, we describe a multigrid Fourier algorithm to solve the linear system, derived in the previous section, for the displacement field. This algorithm is quite similar to the one presented in [16] but there are differences, first it was extended to a multicomponent system, second the incorporation of the substrate was simplified as was the coarse- graining procedure. In [16], the substrate was removed and replaced by effective forces. Unfortunately, standard SOR (the method of Successive over Relaxation) is unstable when this is done and the resulting system had to be under relaxed to stabilize it. Here, the substrate is handled in more seamless way by using Eq. 23 to supply the boundary conditions. As we shall see this formulation coarse grains nicely resulting in an efficient algorithm.

Multigrid is an efficient method for solving linear systems $\mathbf{A u}+\mathbf{F}=0$ where $\mathbf{A}$ comes from the discretization of a partial differential equation. A good introduction to the idea of a multigrid algorithm can be found in the book by Briggs [3]. Two important tools needed to implement multigrid algorithm are coarse-graining, $\mathrm{CF}$, and prolongation operators, FC. The operator CF takes data from fine grid to a coarse grid; if $\mathbf{u}$ is a vector of length $N$ then CFu will be vector of length $N / 2$. The operator FC takes data from a coarse grid to fine grid, if $\mathbf{u}$ is a vector of length $N$ then $\mathrm{FCu}$ is vector of length $2 N$. If $\mathbf{u}$ is smooth then $\mathbf{u} \approx \mathrm{FC}(\mathrm{CF} \mathbf{u})$. Naturally, there are many different ways to formulate these operators but typically coarse-graining operators are based on averaging and the prolongation operators are based on interpolation. In addition, a multigrid algorithm needs a course grained version of $\mathbf{A}$, denoted as $\mathbf{A}^{(2)}$. If $\mathbf{A}$ is $N \times N$ then $\mathbf{A}^{(2)}$ will be $N / 2 \times N / 2$. One natural way to construct $\mathbf{A}^{(2)}$ is to consider the discretization on a coarser grid.

Our goal is to solve $\mathbf{A u}+\mathbf{F}=0$. Suppose, we have an approximate solution, $\mathbf{u}_{\mathrm{a}}$. In order to assess its accuracy, we can compute the residual: $\mathbf{r}=\mathbf{A} \mathbf{u}_{\mathrm{a}}+\mathbf{F}$. The relationship between the residual and the error, $\mathbf{e}=\mathbf{u}-\mathbf{u}_{\mathrm{a}}$, is

$$
\mathbf{A e}+\mathbf{r}=\mathbf{0}
$$

One could solve the above equation for $\mathbf{e}$ and then determine $\mathbf{u}$ using $\mathbf{u}=\mathbf{u}_{\mathrm{a}}+\mathbf{e}$, at first sight that would appear as much work as solving the original system. The crucial observation used by multigrid methods is that if an iterative solver such as Jacobi, Gauss-Seidal, or SOR is used to obtain the approximate solution then the residual, $\mathbf{r}$, and the error, e, are known to be smooth. Indeed, this is why these methods converge so slowly. Since $\mathbf{r}$ is smooth, little information is lost because of coarse-graining. For this reason multigrid methods replace Eq. 30 by

$$
\mathbf{A}^{(2)} \mathbf{e}^{(2)}+\mathbf{r}^{(2)}=0
$$

Once this smaller system is solved the solution then updated using $\mathbf{u}_{\mathrm{a}}^{\text {new }}=\mathbf{u}_{\mathrm{a}}+\mathrm{FC} \mathbf{e}^{(2)}$. One then applies SOR or Jabobi on the fine scale using $\mathbf{u}_{\mathrm{a}}^{\text {new }}$ as a guess. This procedure is repeated until the residual is sufficiently small. This represents a two level multigrid scheme. In most implementations this procedure is extended to many levels.

Implementation of a multigrid method for the system given by Eqs. 15-16 could be accomplished with a standard multigrid method if the film profile was flat and there was no artificial boundary condition. The main difficulty is the formulation of coarse-grained versions of Eqs. 15, 16, and 23. One approach is to use algebraic multigrid methods as in [4]. The approach outlined here was presented in [16] in which the problem was defined on a rectangular domain using fictitious atoms.

\subsection{Fictitious atoms}

We start by defining a rectangular domain. Let $j_{\max }$ be the vertical coordinate of the highest atom. Then, we define our domain of computation to be

$$
\Omega=\{(i, j): \quad 1 \leq i \leq M, \quad 0 \leq j \leq N\} .
$$

where $M$ is the period of the lattice in the horizontal direction and $N>j_{\max }$ is an integer. Some of these sites are occupied and others are not. All sites in $\Omega$ that are not occupied by real atoms are called fictitious atoms. This is illustrated in Fig. 2. 


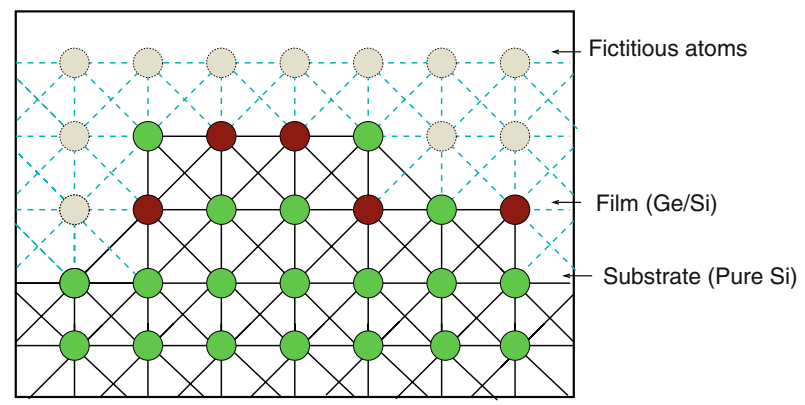

Fig. 2 Computational domain, with real and fictitious atoms. The red circles represent the Ge atoms and the green circles the Si atoms. The null interaction with fictitious atoms is represented by dashed lines

The system of equations in (15) and (16) are now extended to fictitious atoms, simply by setting the connectivity matrix to zero at these sites. This is done by defining the site atom density

$$
p_{i, j}=\left\{\begin{array}{ll}
1 & \text { if }(i, j) \text { is a real atom } \\
0 & \text { if }(i, j) \text { is a fictitious atom }
\end{array} .\right.
$$

With these definitions, the connectivity matrix can be written as

$$
\sigma_{i, j ; m, n}=p_{i, j} p_{i+m, j+n}
$$

In order to simplify the notation, we introduce the following spring strength matrices

$$
\begin{array}{lll}
K_{-1,-1}^{x}=k_{\mathrm{D}} / 2 & K_{0,-1}^{x}=0 & K_{1,-1}^{x}=k_{\mathrm{D}} / 2 \\
K_{-1,0}^{x}=k_{\mathrm{L}} & K_{0,0}^{x}=0 & K_{1,0}^{x}=k_{\mathrm{L}} \\
K_{-1,1}^{x}=k_{\mathrm{D}} / 2 & K_{0,1}^{x}=0 & K_{1,1}^{x}=k_{\mathrm{D}} / 2
\end{array}
$$

and

$$
\begin{array}{lll}
K_{-1,-1}^{y}=k_{\mathrm{D}} / 2 & K_{0,-1}^{y}=k_{\mathrm{L}} & K_{1,-1}^{y}=k_{\mathrm{D}} / 2 \\
K_{-1,0}^{y}=0 & K_{0,0}^{y}=0 & K_{1,0}^{y}=0 \\
K_{-1,1}^{y}=k_{\mathrm{D}} / 2 & K_{0,1}^{y}=k_{\mathrm{L}} & K_{1,1}^{y}=k_{\mathrm{D}} / 2 .
\end{array}
$$

Using these matrices, we can rewrite (15) and (16) as:

$$
\begin{aligned}
& \sum_{m, n=-1}^{1} K_{m n}^{x} \sigma_{\ell j ; m n}\left(\left[u_{\ell+m, j+n}-u_{\ell j}\right]+m n\left[v_{\ell+m, j+n}-v_{\ell j}\right]\right)-F_{\ell j}=0 \\
& \sum_{m, n=-1}^{1} K_{m n}^{y} \sigma_{\ell j ; m n}\left(\left[v_{\ell+m, j+n}-v_{\ell j}\right]+m n\left[u_{\ell+m, j+n}-u_{\ell j}\right]\right)-G_{\ell j}=0 .
\end{aligned}
$$

\subsection{Coarsening and prolongation operations}

A key ingredient in the multigrid technique are the coarsening and prolongation operations used to map data between coarser and finer grids. We let $L=1,2, \ldots L_{\mathrm{g}}$ denote the scale of the grid. $L=1$ denoted the finest scale and $L_{\mathrm{g}}$ the coarsest. In our computations, we will choose the number of grid points in the horizontal direction on the finest scale to be $M=2^{P}$ where $P$ is an integer. Clearly then, $L_{\mathrm{g}} \leq P$. The number grid points in the horizontal direction for the other levels are

$$
M_{L+1}=\frac{M_{L}}{2} \quad L=1,2, \ldots, L_{\mathrm{g}}-1
$$




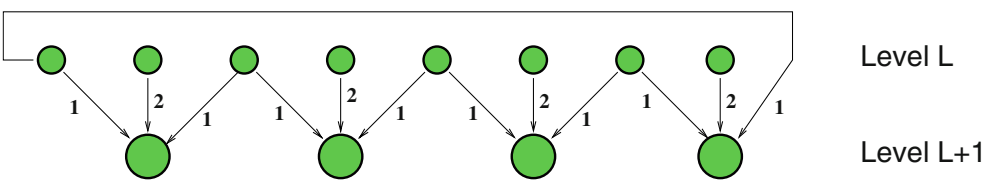

Fig. 3 Coarsening operation in the horizontal direction with even number of grid points. The same relative weights are used in the prolongation, when interpolating values from the coarse to the fine grid.

where $M_{1}=M$. In the vertical direction, we let $N$ denoted the number of grid points on the finest scale. The number of grid points for the other levels is given by

$$
N_{L+1}=\max \left(\left\lfloor\frac{N_{L}+1}{2}\right\rfloor, 1\right) \quad L=1,2, \ldots, L_{\mathrm{g}}-1
$$

where $\lfloor\cdot\rfloor$ represents the integer part.

\subsubsection{Operators in horizontal direction}

In the case of the horizontal direction, we have periodic boundary conditions and the number of grid points will be even for all levels. The coarse-graining procedure we use is displayed schematically in Fig. 3 and can be expressed as

$$
\mathbf{q}^{L+1}=C_{x} \mathbf{q}^{L}
$$

where $\mathbf{q}^{L}$ is a quantity defined on the fine grid, $\mathbf{q}^{L+1}$ is defined on the coarse grid, and $C_{x}$ is the $M_{L+1} \times M_{L}$ matrix given by

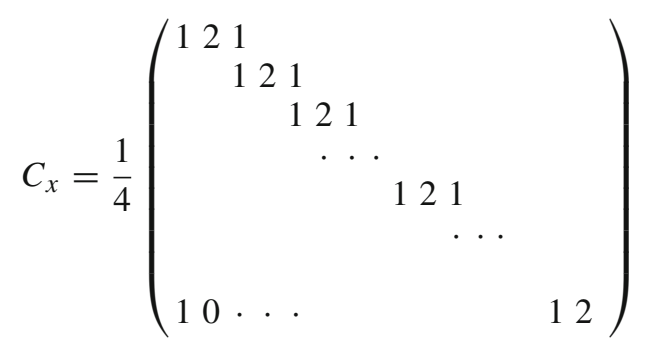

The operation to take variables from the coarse grid to the fine grid is simply

$$
\mathbf{q}^{L}=P_{x} \mathbf{q}^{L+1} \text { where } P_{x}=2 C_{x}^{T}
$$

\subsubsection{Operators in vertical direction}

The situation in the vertical direction is slightly different mainly because the number of grid points, $N_{L}$, can be either even or odd. Our coarse-graining strategy is shown in Figs. 4 and 5. We can write the coarse graining and prolongation operations as follows

$$
\mathbf{q}^{L+1}=C_{y} \mathbf{q}^{L} \quad \text { and } \quad \mathbf{q}^{L}=P_{y} \mathbf{q}^{L+1}
$$




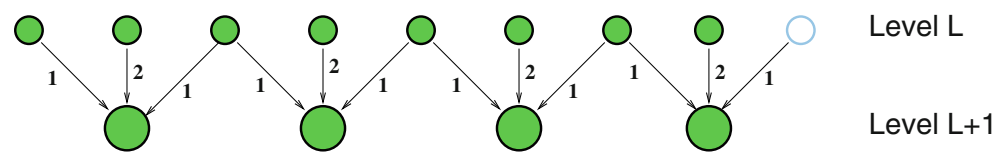

Fig. 4 Coarsening operation in the vertical direction for an even number of grid points on the fine scale. The blue circles represent the grid outside the computational domain where the data is assumed to be zero

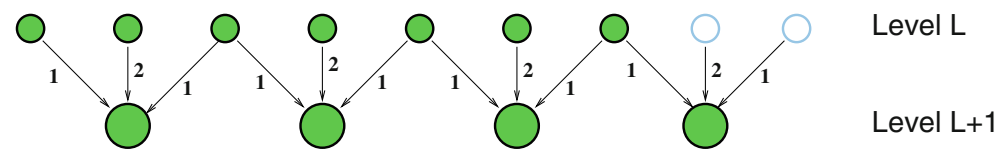

Fig. 5 Coarsening operation in the vertical direction for an odd number of grid points on the fine scale

The matrices $C_{y}$ and $P_{y}$ are defined differently for even and odd $N_{L}$. For $N_{L}$ even we have

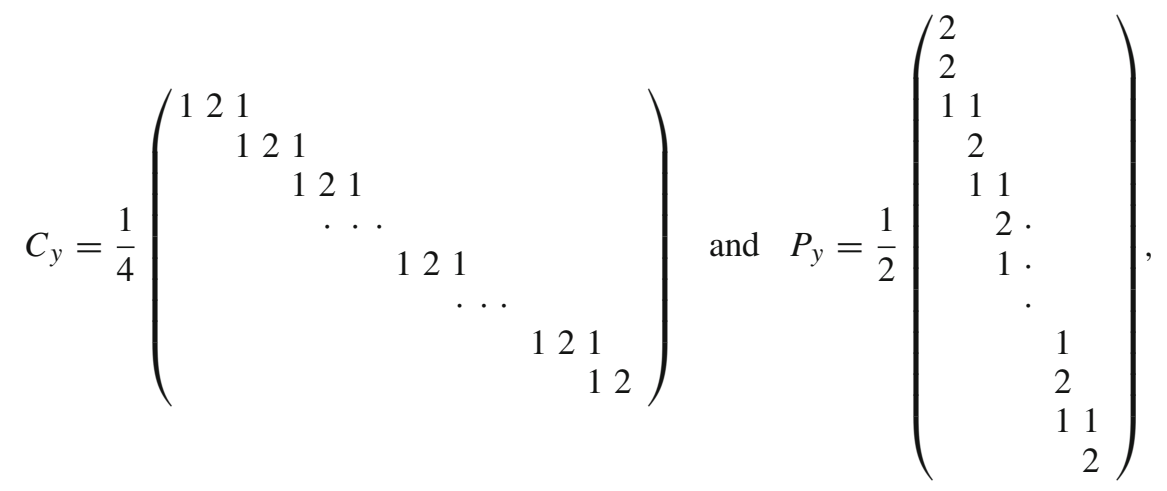

whereas for $N_{L}$ odd we have

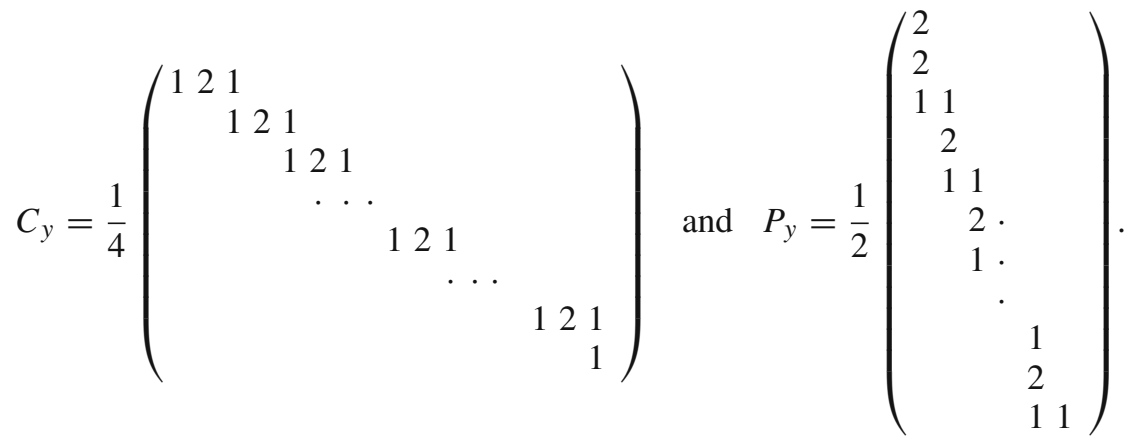

Remark It should be pointed out that $P_{y}$ is not quite equal to $2 C_{y}^{T}$ as was the case in the $x$ direction. In [16], it was incorrectly asserted that their prolongation operator, $P$, could be written as twice the transpose of the coarsening operator.

\subsubsection{Coarsening and prolongation operators in two dimensions}

We construct our coarse from fine operator, CF, and the fine from coarse operator CF, as the tensor product of the one dimensional operators. Namely

$$
\mathrm{CF}=C_{x} \otimes C_{y} \quad \text { and } \quad \mathrm{FC}=P_{x} \otimes P_{y}
$$

Therefore, if $\mathbf{q}^{L}$ is a quantity defined on the grid for level $L$ then we map to the coarser grid of level $L+1$ using

$$
\mathbf{q}^{L+1}=\operatorname{CF}\left(\mathbf{q}^{L}\right)
$$


In an analogous way, we can map $\mathbf{q}^{L}$ to finer grid using

$$
\mathbf{q}^{L-1}=\mathrm{FC}\left(\mathbf{q}^{L}\right)
$$

\subsubsection{Coarse-grained interactions}

Here, it will be outlined how to coarse grain Eqs. 34 and 35. They will be written in terms of the coarsegrained values of the site atom density. The site atom density, $p_{i, j}$, given by (32), is coarsened for all levels $L=2, \ldots L_{\mathrm{g}}$ using CF. The coarse-grained site atom density is used to coarse grain the connectivity matrix as follows

$$
\sigma_{i, j, m, n}^{L}=\sqrt{p_{i, j}^{L}} \sqrt{p_{i+m, j+n}^{L}},
$$

the geometric mean of the site atom densities at the two sites. The coarse-grained version of Eqs. 34 and 35 are written as

$$
\begin{aligned}
& 2^{2-2 L} \sum_{m, n=-1}^{1} c_{\ell j ; m n}^{L} K_{m n}^{x}\left(u_{\ell+m, j+n}^{L}-u_{\ell j}^{L}+m n\left(v_{\ell+m, j+n}^{L}-v_{\ell j}^{L}\right)\right)+F_{\ell j}^{L}=0 \\
& 2^{2-2 L} \sum_{m, n=-1}^{1} c_{\ell j ; m n}^{L} K_{m n}^{y}\left(v_{\ell+m, j+n}^{L}-v_{\ell j}^{L}+m n\left(u_{\ell+m, j+n}^{L}-u_{\ell j}^{L}\right)\right)+G_{\ell j}^{L}=0
\end{aligned}
$$

The factor $2^{2-2 L}$ is typical of coarsened elliptic equations. This can be interpreted as the weakening of springs as several springs are replaced by a single spring. The boundary conditions at $j=-1$ are given by (23) on the corresponding grid level. In other words

$$
\left(\begin{array}{c}
u_{\ell, j}^{L} \\
v_{\ell, j}^{L}
\end{array}\right)=\sum_{\xi=1}^{M_{L}} Q(j) Q^{-1}(0)\left(\begin{array}{c}
\widehat{u}_{\xi 0} \\
\widehat{v}_{\xi 0}
\end{array}\right) e^{i \ell \xi} \text { where }\left(\begin{array}{c}
\widehat{u}_{\xi 0} \\
\widehat{v}_{\xi 0}
\end{array}\right)=\frac{1}{M_{L}} \sum_{\xi=1}^{M_{L}}\left(\begin{array}{c}
u_{\ell, 0} \\
v_{\ell, 0}
\end{array}\right) e^{-i \xi \ell}
$$

The system of equations given by Eqs. 40, 41, and 42, will for sake of convenience, be written as

$$
\mathrm{A}^{L} \mathbf{u}^{L}+\mathbf{F}^{L}=0 .
$$

\subsection{Successive over relaxation}

In our multigrid algorithm, we shall use the method of successive over relaxations to generate approximate solutions. In order to explain our implementation, it is useful to rewrite these equations as

$$
\left(\begin{array}{ll}
a_{x x} & a_{x y} \\
a_{y x} & a_{x x}
\end{array}\right)\left(\begin{array}{c}
u_{\ell j}^{L} \\
v_{\ell j}^{L}
\end{array}\right)=\left(\begin{array}{c}
c_{x} \\
c_{y}
\end{array}\right)-\left(\begin{array}{c}
F_{\ell j}^{L} \\
G_{\ell j}^{L}
\end{array}\right)
$$

where

$$
\begin{gathered}
a_{x x}=2^{2-2 L} \sum_{m, n=-1}^{1} c_{\ell j ; m n} K_{m n}^{x}, \quad a_{x y}=2^{2-2 L} \sum_{m, n=-1}^{1} c_{\ell j ; m n} K_{m n}^{x} m n, \\
a_{y x}=2^{2-2 L} \sum_{m, n=-1}^{1} c_{\ell j ; m n} K_{m n}^{y} m n, a_{y y}=2^{2-2 L} \sum_{m, n=-1}^{1} c_{\ell j ; m n} K_{m n}^{y}, \\
c_{x}=2^{2-2 L} \sum_{m, n=-1}^{1} c_{\ell j ; m n} K_{m n}^{x}\left(u_{\ell+m, j+n}^{L}+m n v_{\ell+m, j+n}^{L}\right),
\end{gathered}
$$


and

$$
c_{y}=2^{2-2 L} \sum_{m, n=-1}^{1} c_{\ell j ; m n} K_{m n}^{y}\left(v_{\ell+m, j+n}+m n u_{\ell+m, j+n}\right) .
$$

We have omitted writing the explicit dependence of the $a$ and $c$ coefficients on $\ell$ and $j$. The relaxation scheme for the above system is based on treating the right hand side as known. Let us denote by $\left(\left(u_{\ell j}^{L}\right)^{k},\left(v_{\ell j}^{L}\right)^{k}\right)^{T}$ the value of the displacement at iteration $k$. Then, the solution at the next iteration, $\left(\left(u_{\ell j}^{L}\right)^{k+1},\left(v_{\ell j}^{L}\right)^{k+1}\right)^{T}$ is computed by relaxing system (44) by one SOR iteration. The SOR relaxation is performed as

$$
\begin{aligned}
\left(u_{\ell, j}^{L}\right)^{*} & =\left(c_{x}^{k}-F_{\ell j}^{L}-a_{x y}\left(v_{\ell j}^{L}\right)^{k}\right) / a_{x x} \\
\left(u_{\ell j}^{L}\right)^{k+1} & =\omega\left(u_{\ell j}^{L}\right)^{*}+(1-\omega)\left(u_{\ell j}^{L}\right)^{k} \\
\left(v_{\ell, j}^{L}\right)^{*} & =\left(c_{y}^{k}-G_{\ell j}^{L}-a_{y x}\left(u_{\ell j}^{L}\right)^{k+1}\right) / a_{y y} \\
\left(v_{\ell j}^{L}\right)^{k+1} & =\omega\left(v_{\ell j}^{L}\right)^{*}+(1-\omega)\left(v_{\ell j}^{L}\right)^{k}
\end{aligned}
$$

where the superscript $k$ on $c_{x}$ and $c_{y}$ indicates that these terms are evaluated using the displacements at the $k$ th iterate.

\subsection{Multigrid V-cycle implementation}

In our computations, we implement the multigrid algorithm using a standard V-cycle which, for sake of completeness, we will now describe. The V-cycle starts with the following steps:

\section{Precomputation}

1. Compute $\mathbf{F}^{1}$ using (10).

2. Compute $N_{L}$ and $M_{L}$ for $L=2$ to $L_{\mathrm{g}}$ using (37) and (36).

3. Coarse grain the site atom density: $p^{L+1}=\mathrm{CF}\left(p^{L}\right)$ for $L=2$ to $L_{\mathrm{g}}$

4. Compute the connectivity matrix: $\left(\sigma_{i, j, m, n}^{L}\right)$ for $L=2$ to $L_{\mathrm{g}}$

5. Initialize first guess for $\mathbf{u}_{\text {guess }}^{1}$ (usually $\mathbf{u}_{\text {guess }}^{1}=0$ or an existing field).

V-cycle

For $L=1$ to $L_{\mathrm{g}}-1$ do the following

Relax $A^{L} \mathbf{u}^{L}+\mathbf{F}^{L}=0$ for $\eta$ steps $(\eta=2$ in our calculations)

Compute residual $\mathbf{r}^{L}=\mathbf{F}^{L}+\mathrm{A}^{L} \mathbf{u}^{L}$

Coarse grain the residual: $\mathbf{r}^{L+1}=\mathrm{CF}\left(\mathbf{r}^{L}\right)$

Set $\mathbf{F}^{L+1}=\mathbf{r}^{L+1}$

Set $\mathbf{u}^{L+1}=0$ (This is the initial condition for the next relaxation)

End Loop

Solve $\mathrm{A}^{L_{\mathrm{g}}} \mathbf{u}^{L_{\mathrm{g}}}+\mathbf{F}^{L_{\mathrm{g}}}=0$ by relaxation

For $L=L_{\mathrm{g}}-1$ to 1 do the following

Prolong the solution on the coarse mesh: $\mathbf{e}^{L}=\mathrm{FC}\left(\mathbf{u}^{L+1}\right)$

Let $\mathbf{u}_{\text {guess }}^{L}=\mathbf{e}^{L}+\mathbf{u}^{L}$

Relax $A^{L} \mathbf{u}^{L}+\mathbf{F}^{L}=0$ with initial guess $\mathbf{u}_{\text {guess }}^{L}$ for $\eta$ steps

End Loop

The accuracy of the solution is measured using the $L_{2}$ norm of relative residual defined as

$$
R_{\text {Global }}=\|\mathbf{r}\|_{2} /\|\mathbf{F}\|_{2}
$$

The V-cycles are repeated until $R_{\mathrm{Global}}<\varepsilon_{\mathrm{G}}$ where $\varepsilon_{\mathrm{G}}$ is a specified tolerance. 
Table 1 Average CPU time in seconds to update the displacement field and calculate the change in elastic energy

\begin{tabular}{llll}
\hline Fourier-multigrid CPU time & & & \\
\hline System size & $\varepsilon_{\mathrm{g}}=10^{-2}$ & $\varepsilon_{\mathrm{g}}=10^{-3}$ & $\varepsilon_{\mathrm{g}}=10^{-4}$ \\
\hline 512 & 0.030 & 0.065 & 0.202 \\
1024 & 0.056 & 0.128 & 0.420 \\
\hline
\end{tabular}

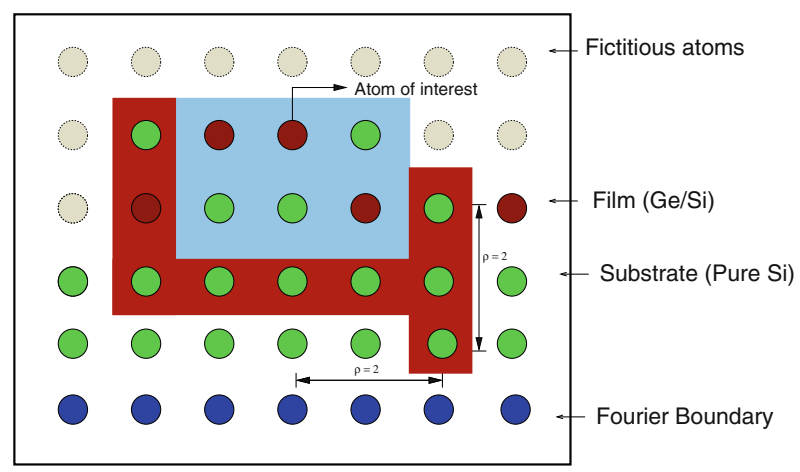

Fig. 6 Computational domain for local elastic solve with $\rho=2$ with real and fictitious atoms. The red circles represent the germanium atoms and the green circles the silicon atoms. The blue region represents the grid points updated by SOR and the red region represents the boundary where $\mathbf{u}=\mathbf{u}_{\rho}$

\subsection{Calculation of elastic energy differences}

Given the surface site, $\left(\ell, h_{\ell}\right)$, our goal is to calculate the change in the elastic energy

$$
\Delta W=W(\mathbf{u})-W\left(\mathbf{u}^{\mathrm{a}}\right)
$$

where $\mathbf{u}$ and $\mathbf{u}^{\mathrm{a}}$ are the displacement fields with and without the atom, respectively. Table 1 shows the computational time required to accomplish this task. In more detail, we consider a film, with 10 monolayers of deposition, having a profile similar to the one shown in Fig. 9. We begin with an updated displacement field and remove an atom and compute the change of elastic energy. The atom is then replaced; this is done each and every surface atom. Results for the computational time are displayed on Table 1 . These were obtained using a 3.6 GHz Intel Pentium 4 Processor Linux Box. It should be pointed out that $\varepsilon_{\mathrm{G}}=10^{-2}$ provides accurate values to $\Delta W$ i.e., within $1-5 \%$.

\section{Local elastic calculations}

Even though the multigrid method greatly reduces the computational time for an update of the elastic displacement field it is still far too slow considering the large number of updates required during the course of a kinetic Monte Carlo simulation. In this section, we will first examine the long-range nature of elastic interactions and then outline a strategy for the computation of elastic energy differences using local updates of the displacement field.

\subsection{Long-range nature of elastic interactions}

As is well known elastic interactions are long ranged and in fact for strained films they are even longer. In order to make this point clear, it is interesting to consider the following quantity

$$
\Delta W_{\rho}=W\left(\mathbf{u} ; \Omega_{\rho}\right)-W\left(\mathbf{u}^{\mathrm{a}} ; \Omega_{\rho}^{\mathrm{a}}\right)
$$

where

$$
\Omega_{\rho}:=\left\{(\ell, m) ; i-\rho \leq \ell \leq i+\rho, h_{i} \geq m \geq h_{i}-\rho\right\}
$$



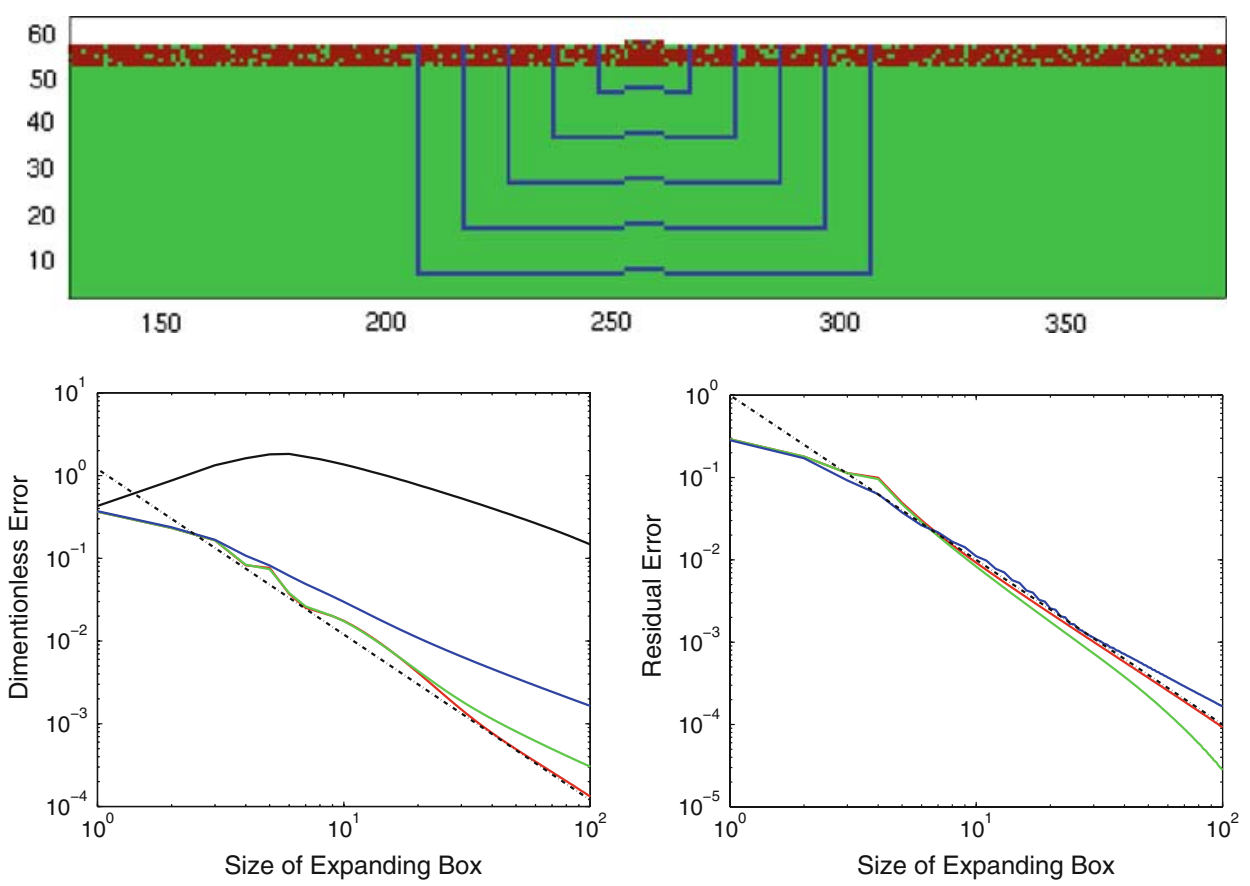

Fig. 7 The upper figure shows a portion of the film along with some of the expanding boxes. The black line shown on the lower left hand figure shows a plot of $\left(\Delta W-\Delta W_{\rho}\right) / \Delta W$ versus $\rho$. The colored lines show plots of $\left(\Delta W-\Delta W_{l o c}\right) / \Delta W$ versus $\rho$. The blue line is for 2 SOR iterations for each box, where green is 10 and red is 50 . The lower right hand figure presents a plot of $R_{\text {loc }}$ for results on the left. In both graphs the dotted line corresponds to $\rho^{-2}$

and

$$
\Omega_{\rho}^{\mathrm{a}}:=\Omega_{\rho} \backslash\left\{\left(i, h_{i}\right)\right\}
$$

Also in the above formula $W\left(\mathbf{u} ; \Omega_{\rho}\right)$ is taken to mean the total elastic energy inside the region $\Omega \rho$ with a displacement field given by $\mathbf{u}$ as shown in Fig. 6. Figures 7 and 8 demonstrate the very slow decay of $\Delta W_{\rho} \rightarrow \Delta W$ by plotting $\left(\Delta W-\Delta W_{\rho}\right) / \Delta W$ versus $\rho$ for two different profiles.

These results can be further understood in the context of continuum elasticity for a film on a semi-finite substrate - which is a reasonable approximation since the ball and spring system is a discretization of linear elasticity.

We take the film profile to be of the form $h(x)=T+c(x)$ where $c(x)$ is a smooth compactly supported function whose region of support includes $x=0$. If one employs the small slope approximation and if a small amount of material is removed from the surface at $(0, h(0))$ then

$$
\Delta W_{\rho}=\Delta W(1+O(T / \rho)) \text { as } \rho \rightarrow \infty
$$

where $\Omega_{\rho}$ is a semi-circular region of radius $\rho$ centered at $(0, h(0))$. For more details see [17].

\subsection{Principle of energy localization}

Given that the goal is to update the displacement field after removing only one surface atom it might seem reasonable to suppose that one could locally update the displacement in the vicinity of the removed atom. However, the above results suggest otherwise. Nevertheless, in [17] the authors present an algorithm to perform local updates to the elastic fields and calculate the change in the elastic energy, with and without the atom, by local calculations. This is based on what is called Principle of Energy Localization [17] which we will now explain.

The idea behind Energy Localization is to compute $\Delta W$ by replacing $\mathbf{u}^{\mathrm{a}}$ by a locally corrected field $\mathbf{u}_{\rho}^{\mathrm{a}}$ which is computed by solving Eqs. 34 and 35 for atoms in the domain $\Omega_{\rho}^{\mathrm{a}}$ using $\mathbf{u}$ as Dirichlet boundary data. 

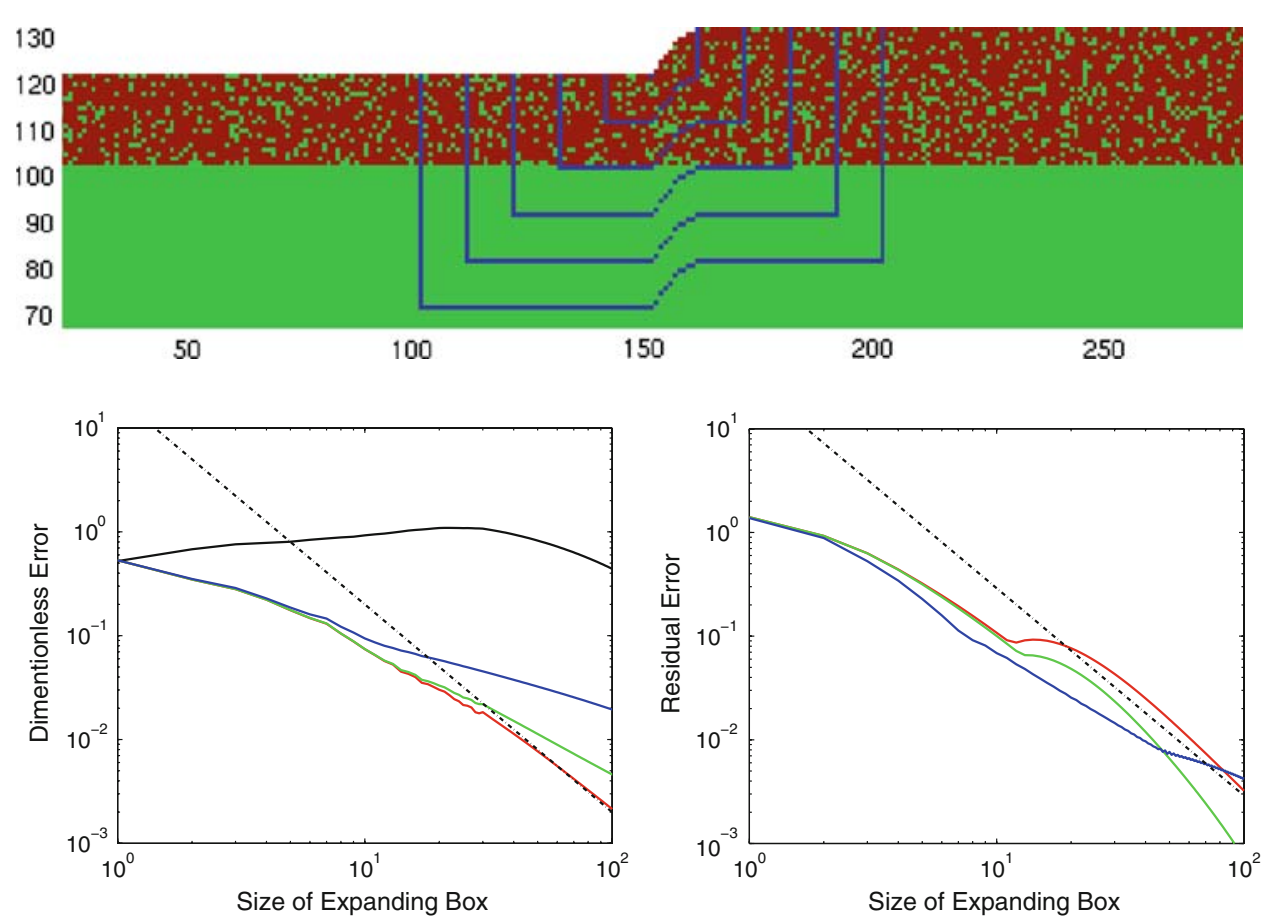

Fig. 8 This is the as Fig. 7 except the film profile is different

In this way, we have the following approximate displacement field for the atom-off configuration

$$
\mathbf{u}^{\mathrm{a}} \approx \begin{cases}\mathbf{u}_{\rho}^{\mathrm{a}} & \text { for }(\ell, j) \in \Omega_{\rho}^{\mathrm{a}} \\ \mathbf{u} & \text { otherwise }\end{cases}
$$

The principle of energy localization states that

$$
\Delta W_{\text {loc }}=W\left(\mathbf{u} ; \Omega_{\rho}\right)-W\left(\mathbf{u}_{\rho}^{\mathrm{a}} ; \Omega_{\rho}^{\mathrm{a}}\right)
$$

will be very close to $\Delta W$ provided that (48) is a good approximation to the displacement field. This result is rather surprising given that $\Delta W_{\rho}$ is a terrible approximation and the only difference between them is that $\Delta W_{\text {loc }}$ uses a approximation to $\mathbf{u}^{\mathrm{a}}$.

In order to assess the accuracy of (48), we note that since both $\mathbf{u}$ and $\mathbf{u}_{\rho}^{\mathrm{a}}$ satisfy Eqs. 34 and 35 exactly for points not on the boundary of $\Omega_{\rho}^{\mathrm{a}}$ then residual will be zero everywhere except for these points. In other words, the atoms on the boundary of $\Omega_{\rho}^{\mathrm{a}}$ experience a small force. With this in mind we define local relative residual in the region $\Omega_{\rho}$ as

$$
R_{\mathrm{loc}}=\frac{1}{\epsilon a_{\mathrm{ss}} k_{\mathrm{L}}} \max _{(\ell, j) \in \Omega_{\rho+1}^{\mathrm{a}}}\left|\mathbf{r}_{\ell, j}\right|
$$

This formula also considers the possibility that the solution inside $\Omega_{\rho}^{\mathrm{a}}$ may not satisfy Eqs. 34 and 35 exactly.

The following numerical results are consistent with the principle of energy localization

- $\Delta W_{\text {loc }}$ is an excellent approximation to $\Delta W$ as $\rho$ increases.

- The local residual $R_{\text {loc }}$ decreases as $\rho$ increases.

Figures 7 and 8 show plots of the relative error, $\left(\Delta W-\Delta W_{\text {loc }}\right) / \Delta W$ for two different profiles. The results show that the method can provide accurate values for the change in elastic energy using local calculations. 

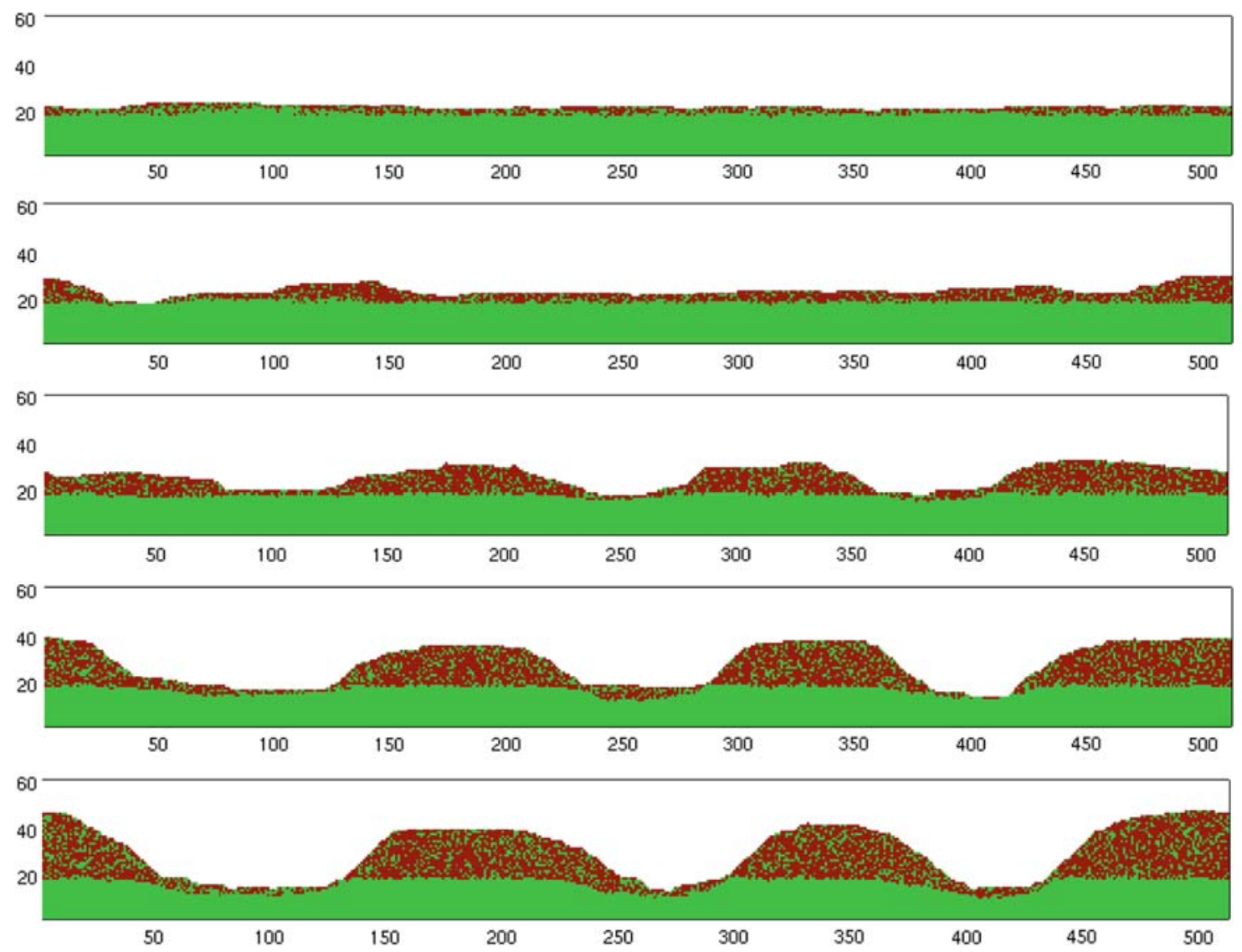

Fig. 9 Pure Ge on Si: The Ge atoms are represented by red squares and the Si by green squares. The results show the evolution after 2, 4, 6, 8 and 10 monolayers deposition

In addition, one can appeal to continuum theory as discussed above to gain further insight into the results discuss above. In the same setting for Eq. 47 it is established in [17] that

$$
R_{\text {loc }}=O\left(\rho^{-2}\right) \text { as } \rho \rightarrow \infty
$$

and that

$$
\Delta W_{\text {loc }}=\Delta W\left(1+O\left(\rho^{-2}\right)\right) \text { as } \rho \rightarrow \infty
$$

\subsection{Expanding box method}

Based on the principle of energy localization, Schulze and Smereka [17] proposed the Expanding Box Method to construct a local update of the elastic displacement field that can be used to find an accurate value for the energy differences. This method is based on using SOR in small neighborhood of the site where the atom was removed. When a change is made to the crystal configuration at one site, few iterations of SOR have negligible impact on the solution at sites that are at a distance of more than one lattice spacing away. The expanding box method constructs a series of nested domains $\Omega_{\rho}^{\mathrm{a}}$ for $\rho=2,3, \ldots, \rho_{\max }$ and finds a locally corrected atom-off displacement field, $\mathbf{u}_{2}^{\mathrm{a}}$. Two iterations of the SOR algorithm described in Sec. 5.3 are used and the atom-on displacement field, $\mathbf{u}$, is used as Dirichlet data for the boundary points of $\Omega_{2}^{\mathrm{a}}$. If it is determined that $R_{\text {loc }}<\varepsilon_{\text {loc }}$, then our local update of the displacement field has been successful. If not we repeat the above procedure until $R_{\mathrm{loc}}<\varepsilon_{\mathrm{loc}}$. If $\rho=\rho_{\max }$ is reached before a successful local update has been achieved we resort to a global update using the multigrid-Fourier method.

In order to give some idea on the computation speed of this method, we present some results in Table 2 for $\rho_{\max }=50$. Results for $\varepsilon_{\mathrm{loc}}=10^{-4}$ were not included since in this case the method fails for almost all 
Table 2 Average CPU time in seconds to update the displacement field and calculate the change in elastic energy

\begin{tabular}{llr}
\hline Expanding box CPU time & & \\
\hline System size & $\varepsilon_{\mathrm{g}}=10^{-2}$ & $\varepsilon_{\mathrm{g}}=10^{-3}$ \\
\hline 512 & $0.0022(466)$ & $0.0176(301)$ \\
1024 & $0.0022(930)$ & $0.0106(608)$ \\
\hline
\end{tabular}

The number in the parentheses is the number of successful applications of the expanding box method

attempts. It is clear that the method is significantly faster than the Fourier-multigrid method. Two specific examples of this method are shown on Figs. 7 and 8. The first example shows a case where the local update would be successful. In the case shown in Fig. 8 the decay is slower than the former case because we are near the base of a large island. In this example, the expanding box method would fail if $\rho_{\max }<50$. The red curves shown in Figs. 7 and 8 are consistent with the expressions given by Eqs. 49 and 50. In addition, the black curves on these figures clearly demonstrate the non-local nature of the elastic energy and is consistent with Eq. 47.

In the kinetic Monte Carlo algorithm, we use to simulate strain file growth, the removed atom is then moved to a neighboring site. Once the atom is moved the atom-on displacement field, $\mathbf{u}$, must be updated. This is also done with the expanding box method. It should be pointed out that the theorems are valid for an initial solution $\mathbf{u}$ that is exact (in other words a globally computed solution is needed). Nevertheless, in practice this "quilt" of locally corrected solutions is found to be of sufficient accuracy to provide faithful energy differences; this issue is discussed in more detail in [17].

\section{Reduced-rejection kinetic monte carlo}

When using Eq. 3 for parameters of physical interest one finds a wide range of different rates. In this situation, the most efficient way to simulate kinetic Monte Carlo is to use a rejection-free implementation. In this case the hopping rate of every surface atom $R$ needs to be known before an event can occur. Even with the expanding box method, this is too slow for practical simulations. Here, we outline a reduced-rejection approach which reduces the number of elastic computations.

In this method the rates, $R_{\ell}$, are replaced by upper bounds, $R_{\ell}^{\text {up }}$ where $R_{\ell}^{\text {up }} \geq R$ where $R^{\text {up }}$ can be quickly evaluated. Now, the atoms are selected with rates $R_{\ell}^{\text {up }}$. Once an atom is selected the actual rate is computed. In order to compensate for the overestimate in the rate, the selected atom will then hop with probability $R_{\ell} / R_{\ell}^{\text {up }}$.

This introduces the possibility of rejection whose rate depends on how closely $R_{\ell}$ is approximated by $R_{\ell}^{\mathrm{up}}$. It is clear by looking at (3) that if we can extract an appropriate upper bound for the change in elastic energy $\Delta W$ then an upper bound on the rates will follow. By performing a large number of numerical experiments, it has been found that $\Delta W$ can be bounded above as follows

$$
\Delta W \leq C(N) w_{\ell}
$$

where $N$ is the number of occupied neighbors of the $\ell$ th surface atom and $w_{\ell}$ is the energy stored in the springs attached to this atom. The function $C(N)$ was found by extensive experiments in [17] and was verified for a multi-component film with intermixing

$$
C(N)=\left\{\begin{array}{lll}
2.4 & \text { if } \quad N=4 \\
3.5 & \text { if } \quad N>4
\end{array}\right.
$$

It seems remarkable that $C(N)$ is independent of the type of surface atom and type of neighboring atoms. The dependence on those factors is contained in $w_{\ell}$.

The upper bounds are now, in view of Eq.3, given as

$$
R_{\ell}^{\mathrm{up}}=R_{o} \exp \left(\frac{-\gamma N+C(N) w_{\ell}+E_{o}}{k_{\mathrm{B}} T}\right) \text { if } N>3
$$

For $N \leq 3$, we use the actual rates from Eq. 3 . 


\section{The algorithm}

The KMC algorithm is the same as that given in [17] but for the convenience of the reader we present it here. Precomputation

1. Calculate elastic field of crystal using multigrid-Fourier method.

2. Compute $R_{\text {dep }}$ and $R_{\ell}^{\text {up }}$ for $\ell=1$ to $M$ using (51)

\section{Algorithm For evolution}

1. Select an event by choosing a uniformly distributed random number $r \in[0, Z)$, with $Z=R_{\text {dep }}+\sum R_{\ell}^{\text {up }}$. This interval represents an overestimate of the sum of rates for atoms hopping plus the rate of deposition. The event to which $r$ corresponds is located using a binary tree search [2].

2. If the event is a deposition, locally update the height, connection arrays and attempt a local elastic solve; revert to a full elastic solve if the expanding box exceeds size $\rho_{\max }$. Update the rate estimates using (51) in the same region in which the elastic field was updated.

3. If the event selected is a hop, then take into account the elastic effects by computing the actual hopping rate $R_{\ell} \leq R_{\ell}^{\mathrm{up}}$ that depends on the energy difference of the system with and without the atom.

(a) Make a copy of the atom-on displacement field $\mathbf{u}$ in the domain $\Omega_{\rho_{\max }}$. Follow the same procedures in Step 2 to compute the displacement field with the atom removed, $\mathbf{u}_{\rho}^{\mathrm{a}}$ (atom off).

(b) Once the elastic field has been updated (locally or globally as necessary), calculate the energy barrier and actual rate $R_{\ell}$.

(c) Use rejection to decide whether or not to make the move. Note that the atom-off calculation must be performed whether or not this move is made.

(d) If the move is rejected, no change is made to the displacement field. Return to Step 1.

(e) If the move is accepted, a hop is made. Update the displacement field in the vacated position using $\mathbf{u}_{\rho}^{\mathrm{a}}$. Perform a second local/global calculation in the atom's new position thereby updating $\mathbf{u}$.

4. Return to Step 1. One event has been completed

\section{Results}

In this section, we present some results using the parameters from [7] namely, $E_{0}=0.53 \mathrm{eV}, R_{0}=2 D_{0} / a_{\mathrm{ss}}^{2}$, $D_{0}=3.83 \times 10^{13} \AA^{2} / \mathrm{s}, a_{\mathrm{ss}}=2.73 \AA, \epsilon_{\mathrm{gg}}=.04, \epsilon_{\mathrm{sg}}=.02$. The spring constants $k_{\mathrm{L}}=13.85 \mathrm{eV} / \mathrm{a}_{\mathrm{s}}^{2}$ and $k_{\mathrm{D}}=k_{\mathrm{L}} / 2$. These were chosen to model the Ge/Si system. In our simulations, we took the bond strength to be $\gamma=0.37 \mathrm{eV}$, whereas [7] choose $\gamma=0.4 \mathrm{eV}$. This was done in order to promote the effects of intermixing. It should be pointed out that the above values are very approximate and our choice of $.37 \mathrm{eV}$ instead of $.4 \mathrm{eV}$ is still well within the range of physically reasonable values. The simulations presented here were conducted at a temperature of $600 \mathrm{~K}$ and we take $\varepsilon_{\mathrm{G}}=\varepsilon_{\text {loc }}=10^{-2}$. Finally, we mention that for results in Fig. 9 the rejection rate was $0.2 \%$ at 1 monolayer and increased to $1.14 \%$ at 10 monolayers. In addition, the displacement field was updated $9.53 \times 10^{8}$ times of which 1,679 were global updates.

\subsection{Example 1}

Our first example aims to clearly establish the importance of intermixing. To this end we shall present two cases. The first is a simulation of the model as discussed in Sect. 2. In the second case, we shall perform the exact same simulation except the Silicon atoms are not allowed to hop: intermixing thereby is prevented. In this way, the importance of intermixing can be readily observed. The results are shown in Figs. 9 and 10. In both cases, pure Germanium was deposited on pure Silicon substrate at a rate of 0.8 monolayers/s.

In the intermixing case (Fig. 9), it is clear that after 2 monolayers islands have not yet formed, one has a thin film composed of mixture of both Silicon and Germanium. When 4 monolayers have been deposited there are indications that islands have formed and after 6 monolayers of deposition there are three distinct islands that persist after further deposition. The case with no intermixing (Fig. 10), is quite different: well defined islands are apparent at 2 monolayers of deposition. These observations can be summarized by a plot of the square of the roughness $\omega^{2}$ (the variance of the height function) as the function of the film thickness in Fig. 11. The data represent the ensemble average over 10 different runs. It can be observed that intermixing initially 


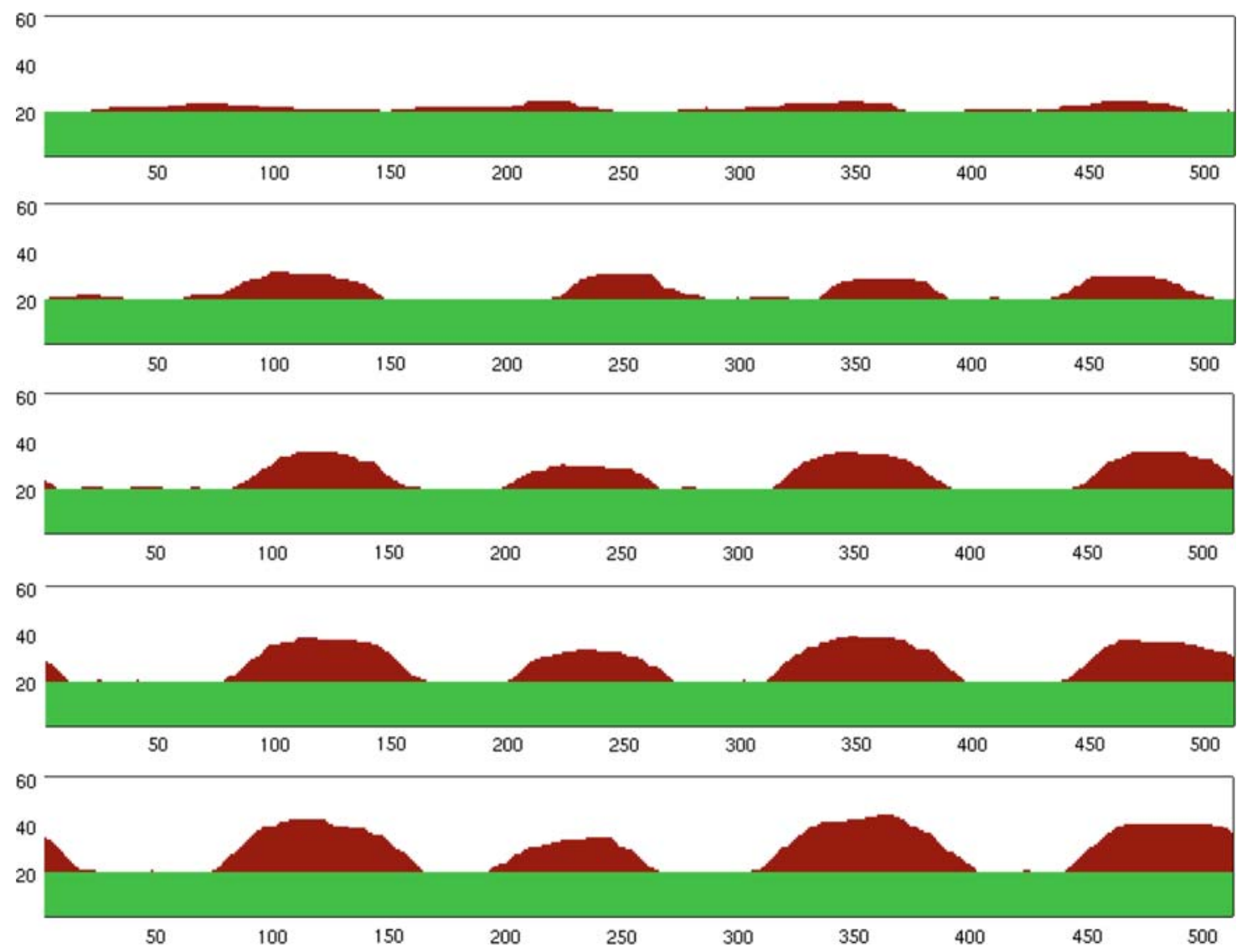

Fig. 10 Pure Ge on Si: The Ge atoms are represented by red squares and the Si by green squares. The results show the evolution after 2, 4, 6, 8 and 10 monolayers of deposition. The Si atoms have been fixed by setting their hopping rates to zero
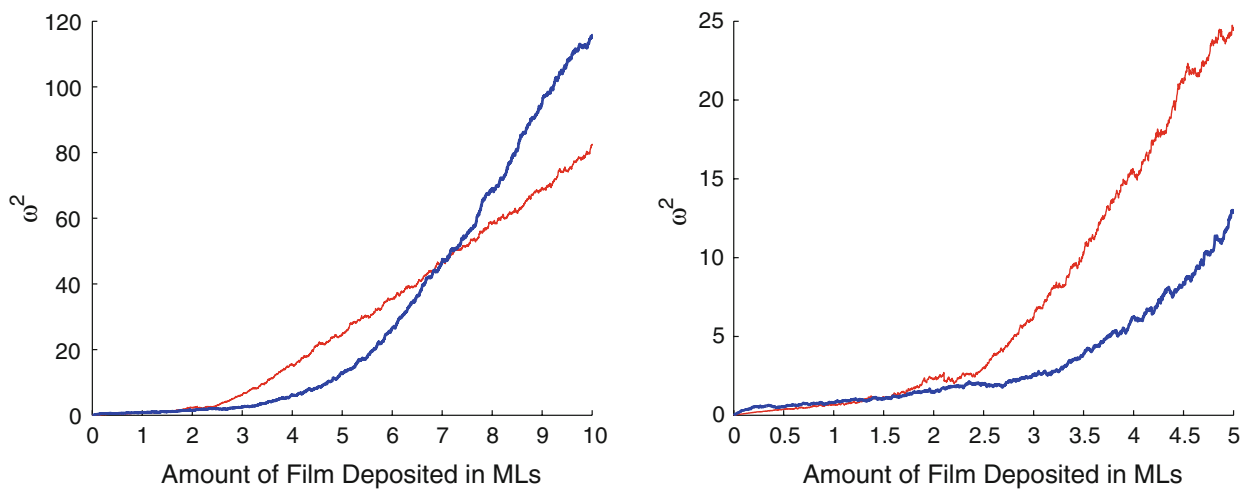

Fig. 11 Roughness plot: this figure shows the plot of $\omega^{2}$ the square of the roughness against the film thickness in monolayers, averaged over 10 independent runs. The red plot shows the behavior with intermixing and the blue without intermixing. The figure on the right zooms into the first five monolayers of crystal growth

suppresses growth of the roughness. However, due to the valley formation the roughness in the intermixing case will ultimately exceed that of the no mixing case.

If one defines an apparent critical thickness as the film thickness at which island first become observable then the apparent critical thickness for the intermixing case is close to three monolayers, whereas for the case with no intermixing the apparent critical thickness is approximately one monolayer. Our simulations show that the intrinsic roughness of the surface due to thermal fluctuations combined with adatom diffusion causes the Germanium and Silicon to mix. An inspection reveals that the pure Ge that was deposited is diluted by around $30 \%$. This mixture has a lower elastic energy density than pure Germanium and consequently the growth rate 

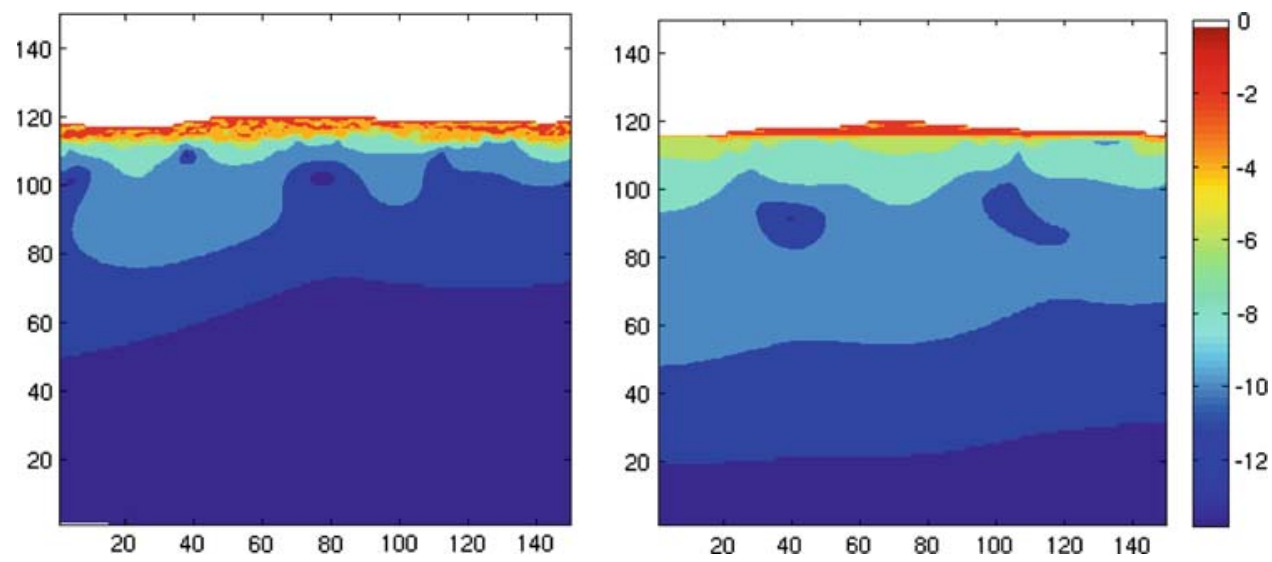

Fig. 12 Elastic energy density plots: the figures show a portion of the elastic energy density (logarithmic scale) for the film after two monolayers of deposition. The figure on the left is the intermixing case and the figure on the right is without intermixing

of islands will be reduced allowing the formation of an apparent critical layer (see Fig. 12). This is consistent with the Asaro-Tiller-Grinfeld (ATG) instability which predicts that the growth rate of the instability is an increasing function of the elastic energy density.

This delay in the onset of the instability is a kinetic effect hence the film is only kinetically stabilized and islands eventually form due to elastic interactions. It also shows the formation of valleys between islands. The valleys arise due stress concentration at the base of the islands. The elastic energy is lowered when the valleys form. It should be noted that the notion of an apparent critical thickness and its connection to the ATG in stability was discussed in [20]. The effect of intermixing on the apparent critical thickness was discussed in the framework of a continuum model in [22].

Figure 13 shows the elastic energy density in the two scenarios at 10 monolayers of deposition. In the case without intermixing stress concentration is observed where the island meets the substrate, whereas with intermixing, the concentrations occur in the valleys between islands. Furthermore, it is observed that the rate of decay of the elastic energy density substrate decays faster in the case with intermixing, see Fig. 14 which plots the elastic energy density along two different cross sections. It is observed that the rate of decay of the elastic energy density substrate decays faster in the case with intermixing. It is somewhat paradoxical that net effect of the intermixing results in a higher energy density in the islands despite lowering it in the early stages of growth.

Equally surprising is that the intermixed crystal has a greater total elastic energy than the crystal without intermixing: the total elastic energy with and without intermixing is $9.363 \times 10^{2} k_{\mathrm{B}} T$ and $5.727 \times 10^{2} k_{\mathrm{B}} T$, respectively. Since all the bond strengths are the same, the chemical energy does not enhance intermixing or segregation. Therefore, we conclude that the intermixing is driven by entropy. We conjecture, despite the larger elastic energy, that the free energy of the intermixing case is less than the case without intermixing.

\subsection{Example 2}

In this example, we deposited 15 monolayers of pure Ge on Si at a rate of 10 monolayers/s and at a temperature of $600 \mathrm{~K}$. This was capped by 30 monolayers of $\mathrm{Si}$ at the same flux and followed by another 15 monolayers of $\mathrm{Ge}$ and so on. The Ge readily forms islands on the Si substrate. When a new Ge film is grown on the capping layer the quantum dots align with the ones buried below. By this process they self assemble to form an array of stacked quantum dots as shown in Fig. 15.

\section{Summary}

In this study, we have presented a kinetic Monte Carlo model for strained heteroepitaxial growth with intermixing. The model is based on a solid-on-solid, bond counting formulation [19] in which elastic interactions are accounted for with balls and springs on cubic lattice [12,7]. We have also introduced an algorithm for the efficient simulation of this model. This algorithm is based on the study in [15-17] but was extended to 

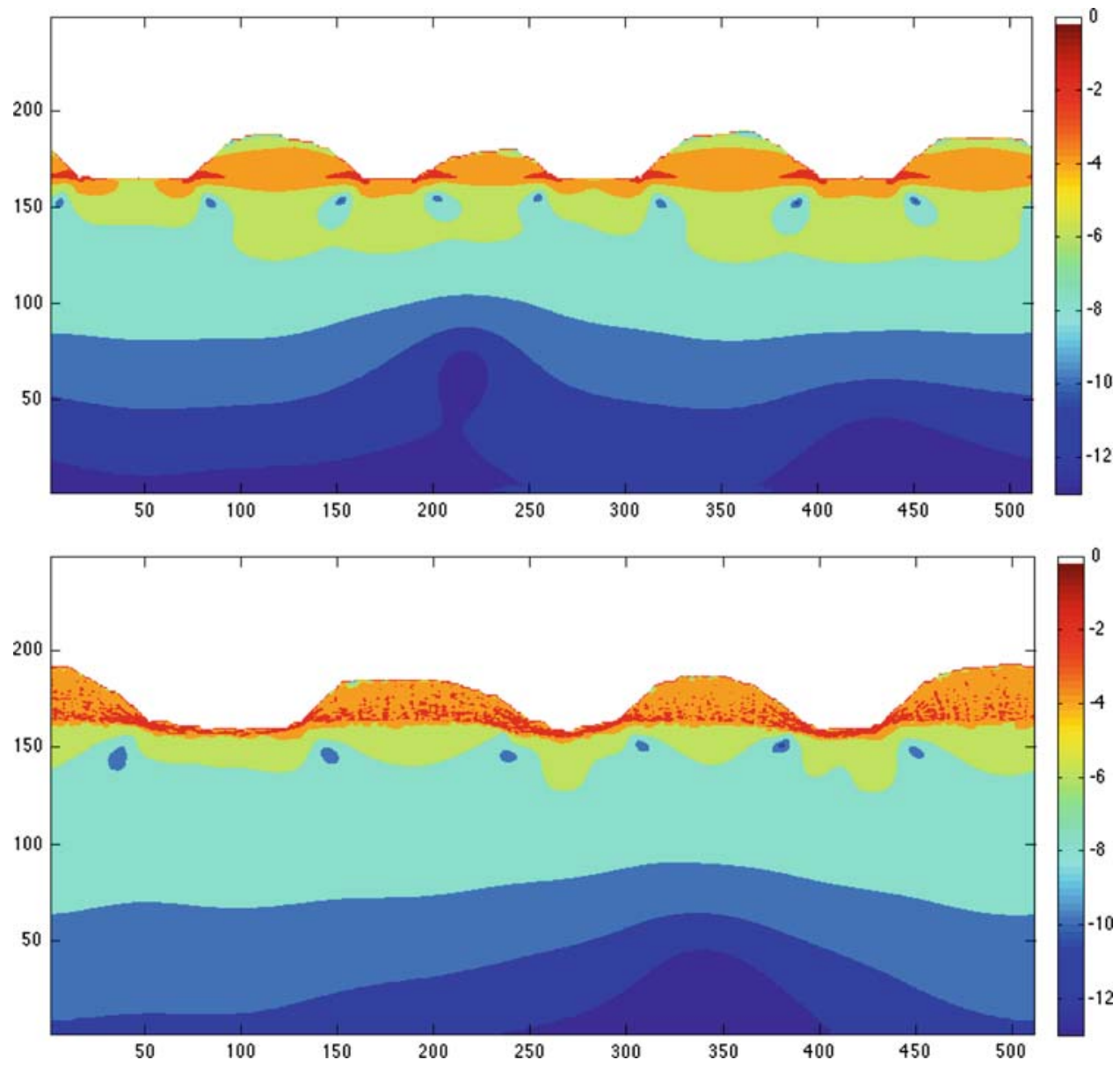

Fig. 13 Elastic energy density plots: the figures show the plots of the elastic energy density (logarithmic scale). The figure on top corresponds to the crystal configuration at 10 monolayers without intermixing shown in Fig. 10 and the bottom one corresponds to the crystal configuration at 10 monolayers with intermixing shown in Fig. 9
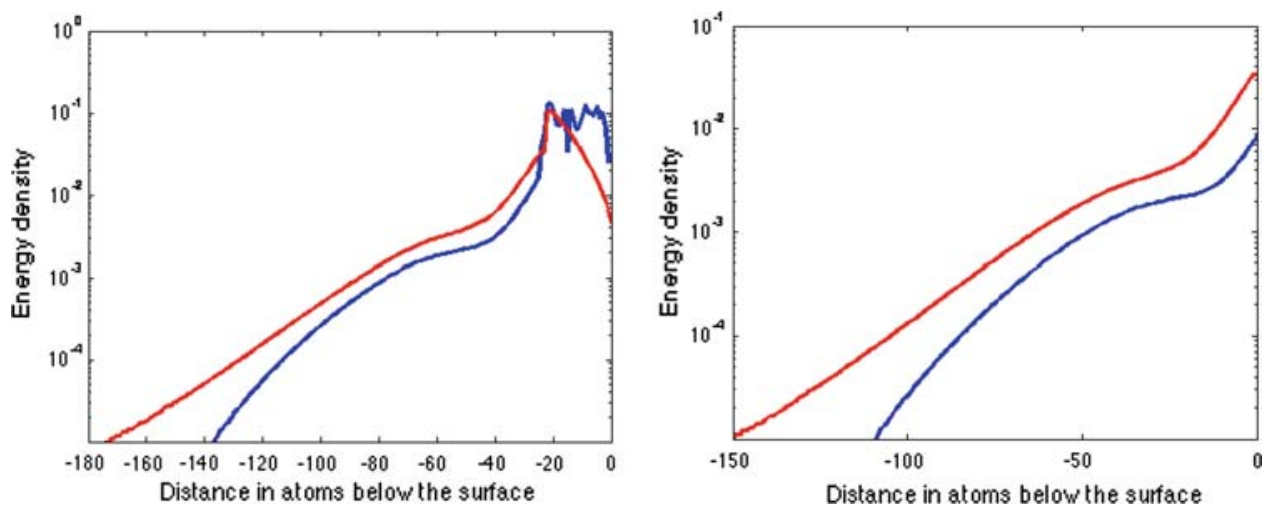

Fig. 14 Elastic energy density plot (cross section): this figure shows the elastic energy density as a function of the distance (in atoms) below the surface. The figure of the left represents a cross section in the center of an island and the one on the right the cross section between two islands. The red color represents the case without intermixing and blue with intermixing.

include intermixing and other improvements. In particular, the Fourier-multigrid method developed in this study has much neater way of coupling the film and the substrate as compared to that in [16]. In addition, the course-graining and prolongation operators have been simplified. We have presented numerical evidence that the principle of energy localization is valid in the case of intermixing and that the asymptotic results presented in [17] also remain true. Our results also indicate that the expanding box method works well in the case of 


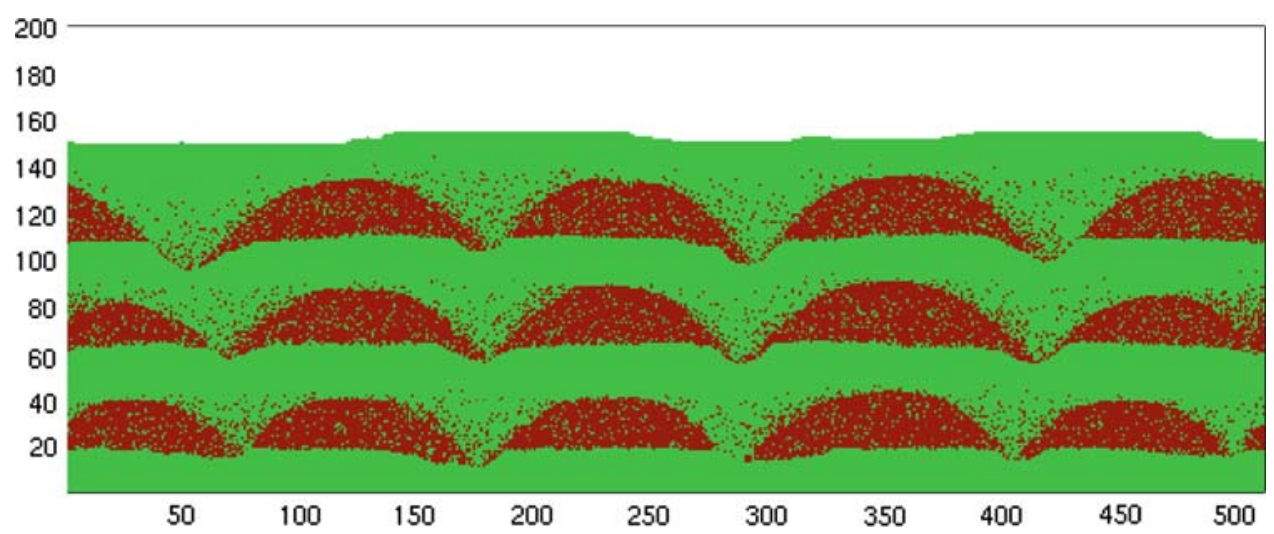

Fig. 15 Stacked quantum dots: quantum dots of 15 monolayers Ge with a capping layer of 30 monolayers $\mathrm{Si}$

intermixing. We have also demonstrated that the formula for the upper bounds on the rates proposed by Schulze and Smereka [17] appears to work well even for mixtures.

A preliminary study on the growth of strained films with intermixing is presented. The study indicates the presence of Asaro-Tiller-Grinfeld (ATG) type instability of the flat strained film leading to the formation of quantum dots. The intermixing is found to initially lower the strain in the film resulting in a stabilizing effect that delays the onset of islands. This gives rise an apparent critical thickness. Eventually, this critical layer disappears as the valleys form. A detailed study of the effects of intermixing on the critical thickness is currently under way. The model also successfully predicts self assembly of stacked arrays of quantum dots.

Acknowledgments We thank Len Sander and Tim Schulze for helpful conversations. This study was supported in part by the following grants from the National Science Foundation, DMS-0553487, DMS-0509124, and DMS-0810113.

Open Access This article is distributed under the terms of the Creative Commons Attribution Noncommercial License which permits any noncommercial use, distribution, and reproduction in any medium, provided the original author(s) and source are credited.

\section{References}

1. Asaro, R.J., Tiller, W.A.: Interface morphology development during stress corrosion cracking: Part I. Via surface diffusion. Metall. Trans. B. 3, 1789-1796 (1972)

2. Blue, J.L., Beichl, I., Sullivan, F.: Faster Monte Carlo simulations. Phys. Rev. E. 51, 867-868 (1995)

3. Briggs, M.W.L.: A Multigrid Tutorial. SIAM, Philadelphia (1987)

4. Caflsich, R.E., Lee, Y.J., Shu, S., Xiao, Y.X., Xu, J.: An application of multigrid methods for a discrete elastic model for epitaxial systems. J. Comp. Phys. 219, 697-714 (2006)

5. Cullis, A.G., Norris, D.J., Walther, T., Migliorato, M.A., Hopkinson, M.: Stranski-Krastanow transition and epitaxial island growth. Phys. Rev. B. 66, 081305 (2002)

6. Grinfeld, M.A.: The stress driven instability in elastic crystals: mathematical models and physical manifestations. J. Nonlinear Sci. 3, 35-83 (1993)

7. Lam, C.H., Lee, C.K., Sander, L.M.: Competing roughening mechanisms in strained heteroepitaxy: a fast kinetic Monte Carlo study. Phys. Rev. Lett. 89, 16102 (1-4) (2002)

8. Lee S., Caflsich R.E., Lee Y.J.: Exact artifical boundary conditions for continuum and discrete elasticity. SIAM J. Appl. Math. 66, 1749-1775 (2006)

9. Lita, B., Goldman, R.S., Phillips, J.D., Bhattacharya, P.K.: Nanometer-scale studies of vertical organization and evolution of stacked self-assembled InAs/GaAs quantum dots. Appl. Phys. Lett. 74, 2824-2826 (1999)

10. Millunchick, J.M., Twesten, R.D., Follstaedt, D.M., Lee, S.R., Jones, E.D., Zhang, Y., Ahrenkiel, S.P., Mascarenhas, A.: Lateral composition modulation in AlAs/InAs short period superlattices grown on InP(001). Appl. Phys. Lett. 70, 1402-1404 (1997)

11. Niu, X., Vardavas, R., Caflisch, R.E., Ratsch, C.: Level set simulation of directed self-assembly during epitaxial growth. Phys. Rev. B. 74, 193403 (2006)

12. Orr, B.G., Kessler, D.A., Snyder, C.W., Sander, L.M.: A model for strain-induced roughening and coherent island growth. Europhys. Lett. 19, 33-38 (1992)

13. Politi, P., Grenet, G., Marty, A., Ponchet, A., Villain, J.: Instabilities in crystal growth by atomic or molecular beams. Phys. Rep. 324, 271 (2000)

14. Quek, S.S., Liu, G.R.: Simulation of surface evolution of quantum dot using meshfree approximation. Thin Solid Films 479, 297-309 (2005) 
15. Russo, G., Smereka, P.: Computation of strained epitaxial growth in three dimensions by kinetic Monte Carlo. J. Comp. Phys. 214, 809-828 (2005)

16. Russo, G., Smereka, P.: A multigrid-Fourier method for the computation of elastic fields with application to heteroepitaxy. Multiscl. Model. Simul. 5, 130-148 (2006)

17. Schulze, T.P., Smereka, P.: An energy localization principle and its application to fast kinetic Monte Carlo simulation of heteroepitaxial growth. J. Mech. Phys. Solids (in press) (2009)

18. Shchukin, V.A., Bimberg, D.: Spontaneous ordering of nanostructure on crystal surfaces. Rev. Mod. Phys. 71, 1125 (1999)

19. Smilauer, P., Vvedensky, D.D.: Coarsening and slope evolution during unstable epitaxial-growth. Phys. Rev. E. 52, 14263-14272 (1995)

20. Spencer, B.J., Voorhees, P.W., Davis, S.H.: Morphological instability in epitaxially strained disclocation-free solid films. Phys. Rev. Lett. 67, 3696 (1991)

21. Spencer, B.J., Voorhees, P.W., Tersoff, J.: Morphological instability theory for strained alloy film growth: the effect of compositional stresses and species-dependent surface mobilities on ripple formation during epitaxial film deposition. Phys. Rev. B. 64, 235318 (2001)

22. Tu, Y., Tersoff, J.: Origin of apparent critical thickness for island formation in heteroepitaxy. Phys. Rev. Lett. 93, $216101(2004)$

23. Tu, Y., Tersoff, J.: Coarsening, mixing, and motion: the complex evolution of epitaxial islands. Phys. Rev. Lett. 98, Art. No. $096103(2007)$

24. Walther, T., Hopkinson, M., Cullis, A.G.: Observation of vertical and lateral Ge segregation in thin undulating SiGe layers on Si by electron energy-loss spectroscopy. Appl. Phys. Lett. 71, 809-811 (1997)

25. Walther, T., Cullis, A.G., Norris, D.J., Hopkinson, M.: Nature of the Stranski-Krastanow transition during epitaxy of InGa on GaAs. Phys. Rev. Lett. 86, 2381-2384 (2001)

26. Wise, S.M., Lowengrub, J.S., Kim, J.S., Johnson, W.C.: Efficient phase-field simulation of quantum dot formation in a strained heteroepitaxial film. Superlattices Microstruct. 36, 293-304 (2004)

27. Wise, S.M., Lowengrub, J.S., Kim, J.S., Thornton, K., Voorhees, P.W., Johnson, W.C.: Quantum dot formation on a strain-patterned epitaxial thin film. Appl. Phys. Lett. 87, Art. No. 133102 (2005) 